\title{
Microvesicles derived from human umbilical cord mesenchymal stem cells enhance alveolar development and attenuate lung inflammation in a rat model of bronchopulmonary dysplasia induced by antenatal lipopolysaccharide
}

Ou Zhou ( $\sim$ zhouou1109@163.com )

Chongqing Medical University Affiliated Children's Hospital https://orcid.org/0000-0001-8868-4651

Jingyi You

Chongqing Medical University Affiliated Children's Hospital

Xiaochuan Xu

Chongqing Medical University Affiliated Children's Hospital

Jiang Liu

Chongqing Medical University Affiliated Children's Hospital

Huijun Qiu

Chongqing Medical University Affiliated Children's Hospital

Chang Hao

Chongqing Medical University Affiliated Children's Hospital

Wenjing Zou

Chongqing Medical University Affiliated Children's Hospital

\section{Wenjie Wu}

Chongqing Medical University Affiliated Children's Hospital

\section{Zhou Fu}

Chongqing Medical University Affiliated Children's Hospital

Daiyin Tian

Chongqing Medical University Affiliated Children's Hospital

Lin Zou

Shanghai Jiaotong University Children's Hospital: Children's Hospital of Shanghai

\section{Research Article}

Keywords: Microvesicles, Mesenchymal stem cells, Bronchopulmonary dysplasia, Antenatal inflammation, Cell proliferation 
Posted Date: January 25th, 2022

DOI: https://doi.org/10.21203/rs.3.rs-798865/v2

License: (c) (i) This work is licensed under a Creative Commons Attribution 4.0 International License. Read Full License 


\section{Microvesicles derived from human umbilical cord mesenchymal stem cells enhance alveolar development and attenuate lung inflammation in a rat model of bronchopulmonary dysplasia induced by antenatal lipopolysaccharide}

Ou Zhou ${ }^{1}$, Jingyi You ${ }^{1}$, Xiaochuan Xu ${ }^{1}$, Jiang Liu' ${ }^{1}$, Huijun Qiu${ }^{1}$, Chang Hao', Wenjing Zou', Wenjie Wu ${ }^{3}$, Zhou Fu', Daiyin Tian ${ }^{1 *}$, Lin Zou ${ }^{1,2,4^{*}}$

${ }^{1}$ Department of Respiratory Medicine, Children's Hospital of Chongqing Medical University, National Clinical Research Center for Child Health and Disorders, Ministry of Education Key Laboratory of Child Development and Disorders, Chongqing Key Laboratory of Pediatrics, Chongqing Engineering Research Center of Stem Cell Therapy, Chongqing 400014, China

${ }^{2}$ Center of Clinical Molecular Medicine, Children's Hospital of Chongqing Medical University, Chongqing 400014, China

${ }^{3}$ Department of Pediatrics, Chongqing Youyoubaobei Women and Children's Hospital, Chongqing 401122, China

${ }^{4}$ Clinical Research Unit, Children's Hospital of Shanghai Jiaotong University, Shanghai 200062, China

\section{* Correspondence:}

Corresponding Author: Lin Zou 
Clinical Research Unit, Children's Hospital of Shanghai Jiaotong University, Shanghai 200062, China

Email: zoulin@shchildren.com.cn; zoulin74@126.com

Telephone: +8618623121280

Co-corresponding Author: Daiyin Tian

Department of Respiratory Medicine, Children's Hospital of Chongqing Medical University, Number. 136, Zhong Shan 2nd Road, Yuzhong District, 400014,

Chongqing, China

Email: t_dy@163.com

Telephone: +8613883048530 


\section{Abstract}

Although it is known that exosomes derived from human umbilical cord mesenchymal stem cells (hUCMSCs) alleviate hyperoxic lung injury of bronchopulmonary dysplasia (BPD) in animal models, the role of microvesicles (MVs) derived from hUCMSCs in BPD is poorly defined. Furthermore, antenatal inflammation has been linked to high risk of BPD in preterm infants. The purpose of this study was to explore whether MVs derived from hUCMSCs can preserve lung structure and function in an antenatal lipopolysaccharide (LPS)-induced BPD rat model and to clarify the underlying mechanism. We demonstrate that antenatal LPS induced alveolar simplification, altered lung function, and dysregulated pulmonary vasculature. Both hUCMSCs and MVs successfully promoted alveolar development and improved lung function. However, hUCMSCs but not MVs restored the loss of pulmonary microvascular vessels $(<100$ $\mu \mathrm{m})$. Furthermore, MVs were mostly uptaken by alveolar epithelial type II cells (AT2) and macrophages. Compared with the LPS-exposed group, MVs restored the AT2 cell number and SP-C expression in vivo and promoted the proliferation of AT2 cells in vitro. MVs also restored the level of IL-6 and IL-10 in lung homogenate. Additionally, upregulated expression of p-AKT, downregulated expression of PTEN, as well as inhibition of MAPK pathway were observed in MVs-treated BPD rats. Taken together, this study suggests MVs derived from hUCMSCs improve lung architecture and function in an antenatal LPS-induced BPD rat model by promoting AT2 cell proliferation and attenuate lung inflammation; thus, MVs provide a promising therapeutic vehicle for BPD treatment. 
Keywords: Microvesicles; Mesenchymal stem cells; Bronchopulmonary dysplasia; Antenatal inflammation; Cell proliferation

\section{INTRODUCTION}

Bronchopulmonary dysplasia (BPD) is a serious and common chronic lung disease of premature infants. It is caused by the imbalance between lung injury and repair in the developing immature lung[1]. BPD is characterized by stunted alveolar development, dysregulated pulmonary vasculature, altered lung function, and pulmonary hypertension $(\mathrm{PH})[2]$. Despite improvements in perinatal care, the incidence of BPD has not been reduced. BPD remains the most common late morbidity and the most frequent complication of extremely preterm birth[3,4].

The cause of BPD is associated with a wide variety of risk factors[5]. Historically, hyperoxia, invasive mechanical ventilation, and sepsis have been considered the key contributors to $\mathrm{BPD}[6]$. However, with the increasing survival rate of the most extremely preterm infants[7], prematurity and low birth weight have become the strongest risk factors of BPD and they are strongly related to antenatal determinants[815]. Therefore, there has been a growing recognition that the early identification and treatment of preterm infants at high risk of BPD may provide greater opportunities to prevent and alleviate $\mathrm{BPD}[16]$. However, in preclinical studies, the most commonly used animal model for BPD research involves prolonged exposure to postnatal hyperoxia. Several studies have reported that antenatal injection of LPS causes impaired alveolar development and dysregulated vasculature by inducing inflammation 
to mimic features of human $\mathrm{BPD}$, even in the absence of postnatal hyperoxia or mechanical ventilation; thus, antenatal LPS exposure may be a better model to reflect the influence of antenatal factors on BPD[16-18].

Human umbilical cord mesenchymal stem cells (hUCMSCs), with low immunogenicity and easy accessibility, are effective for inflammatory disease treatment and regenerative medicine[19]. In preclinical studies, Our group has previously revealed the beneficial effect of hUCMSCs on alleviating BPD in an animal model of exposure to postnatal hyperoxia[20,21]. In clinical studies, many phase 1 clinical trials have reported preliminary data regarding the safety and potential efficacy of MSCs treatment for lung injury[22,23]. MSCs transplantation in preterm infants at high risk of BPD is safe, with no adverse respiratory, growth, and neurodevelopmental effects[24]. Furthermore, MSCs have been shown to exert their beneficial effects via paracrine mechanisms, and MSC-derived conditioned medium revealed a comparable therapeutic effect[25]. As a major paracrine mediator of MSCs, extracellular vesicles (EVs) — especially exosomes (also called small EVs) — have been reported to attenuate hyperoxia-induced lung injury through treatment mechanisms that include three aspects: anti-inflammatory processes, pro-angiogenesis[26,27], and modulation of lung macrophage phenotype[28]. Besides exosomes, microvesicles (MVs, also called large EVs) - another type of EVs that originate from cell membranes, with sizes ranging from 150 to $1000 \mathrm{~nm}[29]$ - have been reported to mediate the modulation of immune interactions, anti-inflammatory processes, and angiogenesis, considering that they contain proteins, RNA, miRNA, and trophic factors derived from parent cells[30,31]. 
One study reported that human MSC-derived MVs could alleviate lung injury induced by bacterial pneumonia[32]. Another study found that MVs promoted angiogenesis on human umbilical vein endothelial cells in vitro[33]. However, the therapeutic effects of MVs on BPD remain largely unknown.

In this study, in an antenatal LPS-induced BPD rat model, we found that MV derived from hUCMSCs were able to enhance alveolar development by promoting AT2 cell proliferation and ameliorating inflammation. Our findings provide insights into the paracrine effects of MSCs on the antenatal rat model of BPD.

\section{MATERIALS AND METHODS}

\section{Animals}

Sprague-Dawley (SD) rats were purchased from the Experimental Animal Center of Chongqing Medical University and maintained at the Animal Laboratory Center of Pediatrics, Children's Hospital of Chongqing Medical University. All animal procedures and protocols were approved by the Ethics Committee of Children's Hospital of Chongqing Medical University. The animals were housed under controlled temperature $\left(22 \pm 1{ }^{\circ} \mathrm{C}\right)$ with a 12-hour day/night cycle with food and water ad libitum. Neonatal mortality was checked daily.

\section{Intra-amniotic (IA) injections of LPS and antenatal rat model of BPD}

As shown in Figure 1A, pregnant female Sprague-Dawley rats (8- to 10-week old) received IA injections of LPS on day 20.5 of gestation (term=day 22.5 of gestion) in 
accordance with a previous report[34]. Briefly, laparotomy was performed on each dam under general anesthesia with isoflurane inhalation. After anesthesia, amniotic sacs were fully exposed from the abdominal cavity; IA injections were started from the amniotic sac closest to the right ovary and were administered to up to 10 sacs per dam in a counter-clockwise sequence. Pregnant rats were randomly assigned to receive normal saline (NS; $50 \mu \mathrm{L}$ per amniotic sac) for the control group, or LPS (10 $\mu \mathrm{g}$ of Escherichia coli 055: B55 diluted with $50 \mu \mathrm{L}$ NS per sac) for the antenatal BPD model. Two days following IA injections, cesarean section was performed on pregnant rats under general anesthesia. All the rat pups (male and female) in the injected amniotic sacs were delivered and then placed with foster mother rats.

\section{Preparation and identification}

Exosomes-free fetal bovine serum (FBS) was prepared overnight using ultracentrifugation at $4^{\circ} \mathrm{C}, 120000 \mathrm{~g}$ for 12 hours. MVs were harvested from the medium of hUCMSCs, obtained from the Chongqing Stem Cell Engineer Research Center. Briefly, P4-P6 HUCMSCs were seeded at $1 \times 10^{5}$ cells per $100 \mathrm{~mm}$ dish and cultured for 48 hours in 10 mL DF-12 with 10\% exosome-free FBS. The medium was collected and centrifuged at $4^{\circ} \mathrm{C}, 400 \mathrm{~g}$ for 5 minutes and $1500 \mathrm{~g}$ for 10 minutes to remove cells and debris. The supernatant was ultracentrifuged at $18000 \mathrm{~g}$ for 30 minutes to pellet the MVs. The total protein concentration of the MVs was measured using BCA kit (Beyotime, China) as per the manufacturer's recommendations. The isolated MVs were stored at $-80^{\circ} \mathrm{C}$ for later use and characterized by TEM (Hitachi, S-3000N, Japan) 
and Zetasizer Nano S90 (Malvern, UK).

\section{hUCMSCs or MVs administration and tissue collection}

On postnatal day 7 (PN7), as illustrated in Figure 2A, the treatment groups received 40 $\mu \mathrm{L}$ hUCMSCs $\left(1 \times 10^{6}\right.$ cells per pup) or MVs by intratracheal route, while the control groups received $40 \mu \mathrm{L}$ of intratracheally administered NS. On PN14, the pups were anesthetized with an intraperitoneal injection of pentobarbital sodium $(40 \mathrm{mg} / \mathrm{kg})$. Then, the thoracic cavity was opened and the lungs were perfused with cold PBS through the heart. The lungs were harvested for histological assessment. The hearts were weighed to determine RVI. Body weight was measured at birth, on PN7, and on PN14. Animals were randomized into different groups using table of randomized numbers. Treatments were administered in a blinded manner. In order to minimize the cage effect, pups among groups were divided and housed in 4-4 cages, with five to eight pups per cage.

\section{Lung alveolarization assessment}

One 4-mm-thick transverse section was taken from the midplane of the left lobe of the fixed lungs per animal and processed and embedded in paraffin wax. All the sections were stained with hematoxylin and eosin, and alveolarization was assessed by performing radial alveolar counts (RAC), secondary septa count, and median linear intercepts (MLI) as previously described[21].

\section{Pulmonary vasculature assessment}


Pulmonary vasculature was measured by immunofluorescence with von Willebrand factor (vWF). Fixed left lungs were embedded in glue (Sakura, Japan) and cut with a microtome at $10 \mu \mathrm{m}$ at $-20^{\circ} \mathrm{C}$ (Leica CM1950, Germany). Frozen sections and cells were then fixed with 4\% PFA and blocked with $10 \%$ BSA. Subsequently, the samples were stained with anti-vWF antibody (1:100, PA5-80223, Thermo Fisher Scientific, USA), washed, and incubated with goat anti-mouse Fluor cy3-conjugated secondary antibody (Proteintech, USA). Cell nuclei were counterstained with DAPI for 15 minutes, and fluorescence was observed on a Leica laser confocal microscope $(\mathrm{C} 2+$ system, Nikon, Japan).

\section{Lung function and right ventricular hypertrophy}

Lung function was determined in PN14 pups with a computer-controlled small-animal ventilator (Emka, USA). Briefly, the rats were anesthetized with pentobarbital sodium $(40 \mathrm{mg} / \mathrm{kg})$, intubated following tracheostomy, and mechanically ventilated at a rate of 150 breaths/min, with a tidal volume of $8 \mathrm{~mL} / \mathrm{kg}$ and a positive end-expiratory pressure (PEEP) of $3 \mathrm{~cm} \mathrm{H}_{2} \mathrm{O}$ with the computer-controlled small-animal ventilator (Emka, USA). LR and Cdyn were recorded every five seconds. Right ventricular hypertrophy was determined by the right ventricular index (RVI), which represents the weight of right ventricle relative to left ventricle + septum $(\mathrm{RV} /(\mathrm{LV}+\mathrm{S}))$. Briefly, after removing the arterial and adipose tissue on the epicardium, We collected and weighed the left ventricle plus the interventricular septum and the right ventricle tissue by cutting along the edge of the ventricle and the interventricular septum. 


\section{MVs localization in the lungs of antenatal LPS-induced BPD rats}

MVs were labeled with a DiO Green Fluorescent (Beyotime, China) as per the manufacturer's protocol. Immunofluorescence localization of donor MVs was performed on $10-\mu$ m-thick cryostat sections on PN9 (48 hours after MVs administration). The following primary antibodies were used as markers of alveolar epithelial type I cells (AT1), AT2, vascular endothelial cells, vascular pericytes, total macrophages, and smooth muscle cells: Aquaporin-1 (AQP1,1:200, Abcam), pro surfactant protein C (SP-C, 1:100; Abcam), CD31 (1:100, Abcam), NG2 (1:200, Abcam), Iba-1 (1:200, Abcam), and $\alpha$-smooth muscle actin ( $\alpha$-SMA) (1:200; Abcam), respectively. Then, Cy-3 dye-labeled IgG was used as the secondary antibody (Beyotime, China). Fluorescence was observed on Leica laser confocal microscopy (C2+ system, Nikon, Japan), and at least five different visual fields were randomly selected from each sample.

\section{Western blotting}

The lung tissues were harvested in a lysis buffer [25 mMTris- $\mathrm{HCl}(\mathrm{pH} 7.5), 137 \mathrm{mM}$ $\mathrm{NaCl}, 2.7 \mathrm{mM} \mathrm{KCl}, 1 \%$ Triton $\mathrm{X}-100$ ] containing protease and phosphatase inhibitors cocktail (Sigma-Aldrich, St. Louis, MO). The protein concentration was measured using BCA protein assay reagent (Beyotime, China). Equal amounts of proteins were separated using SDS-PAGE and transferred to polyvinylidene difluoride membranes (Thermo Scientific, Rockford, IL). The membranes were blocked with 5\% skim milk 
in PBS containing $0.1 \%$ Tween 20 (PBS-T) for one hour and then incubated with the specified antibodies. Signals were detected using the ECL detection system (Gene Company Limited, Hong Kong, China).

\section{Statistical analysis}

Statistical analysis was performed with the GraphPad Prism software (Version 5.0, San Diego, CA, USA). A $t$-test was used for statistical comparisons between two groups, and a one-way analysis of variance (ANOVA) with Kruskal-Wallis/Dunns post hoc test was applied for significance testing among more than two groups. Investigators were blinded to experimental groups for histological analysis and physiological measurements. Statistical significance is indicated as follows: $* P<0.05, * * P<0.01$, $* * * P<0.001$. NS means no significance. Data are presented as mean \pm standard error.

\section{Results}

\section{Establishment and characterization of an antenatal LPS-induced BPD model}

Considering that antenatal LPS injection could cause impaired alveolar structure and dysregulated vasculature to mimic human BPD features[34], we established the rat model of BPD by IA injection of LPS and cesarean delivery. IA LPS lead to $88 \pm 3 \%$ survival of rat pups on PN1, and no more pups dead on PN7 and PN14. The pups' lungs were collected for analysis on PN1, PN7, and PN14 (Fig. 1A). Compared with the NS control (NS Ctrl), the lung structures in the LPS group had typical characteristics of alveolar simplification (Fig. 1B). The MLI in antenatal LPS without hyperoxia on PN7 
and PN14 was significantly higher than that in the NS group, although without statistical difference on PN1 (Fig. 1C). The secondary septa were significantly reduced in the LPS group compared with the NS group on PN1, PN7, and PN14 (Fig. 1D), demonstrating the successful establishment of the rat model of BPD induced by intraamniotic injections of LPS (IA-LPS BPD model).

\section{hUCMSCs treatment improves lung development and alleviates RVH in IA-LPS BPD model rats}

Given that there is currently no report on the effects of hUCMSCs on IA-LPS BPD model, we first examined the effect of hUCMSCs on the antenatal rat model of BPD induced by antenatal LPS. The pups received hUCMSCs on PN7 by transtracheal administration (Fig. 2A). We found that compared with the rats exposed to LPS alone, the lung development was improved on PN14, the MLI was significantly decreased, and the RAC was increased by hUCMSCs treatment (Fig. 2B-D). Although all the groups had the same birth weight, rats exposed to LPS had a slow weight gain, but there was a catch-up growth period after transtracheal administration of hUCMSCs (Fig. 2E). Small vessels $(<100 \mu \mathrm{m})$ in rats exposed to LPS were significantly less abundant than those in the NS group; however, the abundance of small vessels increased after hUCMSCs treatment, compared with the LPS group (Fig. 2F, 2G). The pulmonary function results showed that hUCMSCs treatment reduced lung resistance, increased lung compliance, and decreased RVH as compared with rats exposed to LPS alone (Fig. 2H-J). 


\section{Characterization of hUCMSCs-derived MVs}

Considering that MVs derived from hUCMSCs have multiple regenerative roles in skin repair and other diseases[30,31], we isolated MVs from hUCMSCs by ultracentrifugation and characterized them by electron microscope observation and nanosizer measurement. According to scanning electron microscopy, the MVs showed a double membrane structure (Fig. 3A), and WB confirmed the expression of MVs markers, CD9, CD63, CD81, TSG101, and Alix (Fig. 3B). Nanosight analysis showed that the diameter of the MVs ranged from 100 to $900 \mathrm{~nm}$, with the main peak at $255 \mathrm{~nm}$ (Fig. 3C). The total protein content harvested from $200 \mathrm{~mL}$ supernatant was $165.2 \pm 5.7$ $\mu \mathrm{g}$ (data not shown). Subsequently, we determined whether MVs can be internalized into the MLE-12 cells (an AT2 cell line). MVs labeled with red fluorescence PKH26 co-localized with the green fluorescence signals of cytoskeleton stained with phalloidin, indicating the internalization of MVs into the AT2 cells (Fig. 3D). We then performed mimic infection injury in vitro using LPS in the following experiments, so the effects of a series of differing LPS concentrations on MLE-12 cells were evaluated. Treatment with LPS at a concentration higher than $160 \mu \mathrm{g} / \mathrm{mL}$ for 48 hours obviously inhibited AT2 proliferation (Fig. 3E). Therefore, $80 \mu \mathrm{g} / \mathrm{mL}$ of LPS was selected for the subsequent in vitro experiments.

MVs improve lung structure and prevent loss of lung function in IA-LPS BPD model 
After characterization of MVs, we next addressed the therapeutic effects of MVs in the IA-LPS BPD model, to determine whether MVs partly mediated the effects of hUCMSCs. Firstly, to determine the optimal MVs concentration, we performed a series of dose-response experiments in vivo. MLI measurement on PN14 revealed that lung recovery was dependent on the concentration of the MVs administrated on PN7, and the peak was $20 \mu \mathrm{g}$. Since there was no significant difference in MLI among $20 \mu \mathrm{g}, 40$ $\mu \mathrm{g}$, or $80 \mu \mathrm{g}$ of MVs, we selected $20 \mu \mathrm{g}$ of MVs for the following in vivo experiments (Fig. 4A, 4B). In the rat lung tissue, antenatal LPS-induced impaired alveolarization was significantly enhanced by transtracheal administration of MVs (Fig. 5A). LPS exposure increased MLI and decreased RAC, but the effects were reverted after MV treatment (Fig. 5B, 5C). Besides, there was a catch-up growth after transtracheal administration of MVs (Fig. 5D). In terms of angiogenesis, however, MVs did not normalize the aberrant loss of small vessels caused by LPS (Fig 5E, 5F). MV treatment prevented loss of lung function, as shown by reduced lung resistance and increased lung compliance (Fig. 5G, 5H). Increased RVH induced by LPS was also reversed by MVs (Fig. 5I).

\section{Duration and localization of MVs in vivo}

After addressing the therapeutic effects of MVs in the IA-LPS BPD model, we proceeded to examine the distribution of transplanted MVs in the lung tissue in vivo. Firstly, MVs were labeled with $\mathrm{DiO}$ green fluorescence and examined by immunofluorescence staining at different time points. The DiO green fluorescence 
gradually increased and reached a maximum at 48 hours post-administration, then gradually decreased and finally disappeared after 96 hours (Fig. S1). Then, colocalization of $\mathrm{DiO}$ green fluorescence with various lung cell markers was examined by immunofluorescence staining at 48 hours (Fig. 6A). DiO green fluorescence most frequently co-localized with SP-C-positive AT2 (18.2\%) and was also identified with AQP-1-positive AT1 (4.5\%), CD31-positive endothelial cells (7.6\%), Iba-1-positive alveolar macrophages (14.0\%), $\alpha$-smooth muscle actin-positive smooth muscle cells (2.4\%), and NG-2-positive pericytes (2.4\%) (Fig. 6B).

\section{MVs increase the number of AT2 cells and attenuate lung inflammation in IA-LPS BPD model rats}

Given that AT2 and lung macrophages were the two major cell types responsible for the uptake of MVs in vivo, we next investigated the effects of MVs on these two target cells. For AT2 cells, immunofluorescence staining of SP-C showed that LPS exposure decreased SP-C(+) cells, whereas MVs restored the number of SP-C $(+)$ cells (Fig. 7A, 7B). We also determined the protein level of SP-C, the major surfactant synthetized by AT2 cells. Antenatal LPS exposure decreased the expression of SP-C, which was partially reversed by MV treatment (Fig. 7C, 7D). SP-A1, SP-B, and SP-D, the other three surfactants synthetized by AT2 cells, were also determined by WB; we found that they also decreased after LPS exposure, but they were not restored by MV treatment (Fig. S2).

Then, macrophage infiltration in the lung tissue was measured by Iba-1 
immunofluorescence staining. Antenatal LPS exposure induced significant macrophage infiltration in the rat lung tissue (Fig. S3). In the treatment group, MVs significantly reduced macrophage infiltration in comparison with the LPS group (Fig. S3). IL-6 (proinflammatory cytokine) and IL-10 (anti-inflammatory cytokine), major inflammation mediators released by macrophages, were measured by ELISA. The concentration of IL-6 was increased, while IL-10 was decreased in lung homogenates of LPS-exposed rats, and these responses were significantly reversed by transtracheal administration of MVs (Fig. 7E, 7F). Taken together, MVs from hUCMSCs have antiinflammatory effects on the IA-LPS BPD model in vivo.

\section{MVs improve MLE-12 cells proliferation following LPS-induced injury in vitro}

Given that AT2 cells are the stem cells in the newborn lung and play a role in the alveolar structure involved in pulmonary respiratory function[35], we further investigated the effects of MVs on MLE-12 cells in vitro. MLE-12 cells were treated by LPS with or without MVs for 48 hours. Then, they were evaluated by Ki-67 and Annexin V/PI staining. Ki-67 staining showed that LPS exposure significantly reduced the proliferation rate of MLE-12 cells, which was improved to normal level by MV treatment (Fig. 8A, 8B). The flow cytometry analysis showed that LPS did not alter the apoptotic rate of MLE-12 cells (data not shown). The CCK-8 assay results further illustrated that MV treatment improved the LPS-induced decrease in MLE-12 cells' survival (Fig. 8C). 
MVs improve alveolarization and attenuate lung inflammation associated with the

\section{PTEN/AKT and the MAPK pathways}

Subsequently, we explored the specific mechanism by which MVs improve alveolarization and attenuate lung inflammation in the IA-LPS BPD rat model. The level of PTEN significantly increased and p-AKT expression remarkably decreased in rat lungs from the IA-LPS BPD rats compared with the control group. MV treatment reversed the protein levels of both PTEN and p-AKT (Fig. 9A, 9B). Meanwhile, the results of WB showed that LPS exposure increased the expression levels of p-p38, pJNK, and p-ERK, while MV treatment partially suppressed p-p38, p-JNK, and p-ERK expression after antenatal LPS exposure (Fig. 9C, 9D). However, the expression of VEGF-A suppressed by antenatal LPS exposure was not restored by MVs administration (Fig. 9E, 9F).

\section{Discussion}

Although there is not yet effective treatment for BPD, the advent of MSCs provides new hope for BPD treatment. Many preclinical studies have demonstrated that MSCs and small extracellular vesicles (or exosomes) have a protective effect on lung injuryon BPD[21,27,36,37]. Furthermore, most clinical trials of MSCs in BPD are at a phase I stage, demonstrating the safety of stem cell therapy in human. However, the therapeutic effects and function of large extracellular vesicles (or MVs) derived from MSCs on BPD are poorly understood.

In this study, we showed that the MVs derived from hUCMSCs enhanced alveolar 
development and alleviated lung inflammation in the IA-LPS BPD model, and this protection was associated with the PTEN/AKT pathway and the MAPK pathway. These findings also suggest that MVs are key paracrine therapeutic mediators of hUCMSCs and show potential for application in safe and effective cell-free therapy for BPD associated with antenatal factors.

Many studies have shown that antenatal factors are strongly associated with susceptibility to BPD. However, the most commonly used animal model for BPD research involves exposure to postnatal hyperoxia; thus, it cannot reflect antenatal factors that influence BPD. Here, we used a rat model of BPD induced by intra-amniotic injections of LPS; LPS induced impaired alveolarization and diminished lung function, particularly mimicking BPD of preterm infants in humans[38,39].

Functions of EVs depend on their ability to interact with recipient cells to deliver their contents (proteins, lipids, and RNAs)[40]. Our data showed that MVs were mainly uptaken by AT2 cells (18.2\%) and macrophages (14.0\%), and were rarely observed in vascular endothelial cells (7.6\%) and vascular pericytes (2.4\%). However, our findings are not fully consistent with the previous report that exosomes were mostly uptaken by vascular pericytes (22.7\%), AT2 cells (19.5\%), and macrophages (21.3\%)[36]. The difference in the cellular uptake ability of exosomes and MVs in vascular pericytes might be related to the difference in the physicochemical properties of these two types of EVs, such as origin, size, morphology, buoyant density, and so on[41].

AT2 cells have critical secretory and regenerative roles in the alveoli to maintain lung 
homeostasis [35]. Our data showed that MVs diminished most of the effects of antenatal LPS-injury on AT2, suggesting similar therapeutic effects to those of exosomes on AT2 in hyperoxia-induced BPD model[21]. Additionally, there are many other lung stem cells, such as bronchioloalveolar stem cells, Clara cells, basal cells, and distal airway stem cells $[42,43]$. One study has reported that MSCs increase bronchioloalveolar stem cells in hyperoxia-induced bronchopulmonary dysplasia[44]. Further studies should investigate the effects of MVs on different lung stem cells.

As another major target cell of MVs, lung macrophages are dominant immune cells in the inflammation[45]. Our results showed that antenatal LPS induced macrophage infiltration and this response was restored by MV treatment, which decreased the proinflammatory factor IL-6 and increased the anti-inflammatory factor IL-10 to normal level. Several studies have noted that pulmonary macrophages occupy an "M2like" phenotype, which can persist for several months in $\operatorname{BPD}[28,46]$. Due to the antiinflammatory action of MVs, further studies should examine whether MVs could modulate the dysregulated macrophages phenotype in an experimental BPD model.

It has been shown that the proliferation of AT2 cells in lung injury is linked to abnormal expression of PTEN/Akt[47]. As a major proliferation-linked signaling pathway, the PTEN/AKT pathway was examined in our study. Our data showed that MV treatment reversed the protein levels of PTEN and p-AKT, suggesting that the AT2 cell proliferation promoted by MVs may related to the PTEN/AKT pathway.

Furthermore, previous studies have shown that the antenatal exposure of preterm infants to infection and inflammation may result in adverse fetal consequences, such as 
$\mathrm{BPD}$ [48]. Activation of MAPK signaling is important in the response to inflammation [49]. The signaling mediators of MAPK include ERKs, JNKs, and p38 MAPK[50]; however, their role in antenatal LPS-induced lung injuries has not been identified. Our results showed that the expression levels of $\mathrm{p}-\mathrm{p} 38$, $\mathrm{p}-\mathrm{JNK}$, and p-ERK were significantly increased, but were suppressed by MV administration. These data suggested that MVs alleviate LPS-induced lung injuries by a mechanism associated with the suppressed MAPK pathway. In addition, MVs were ineffective at promoting the expression of VEGF-A, which might be the reason why MVs did not restore pulmonary microvasculature; however, this aspect deserves further investigation.

Although encouraged by these findings, we acknowledge several limitations of this study. First, the concentration and duration of LPS in ammonitic sac, as well as intake of LPS in the lung, were not examined. Second, we only administered MVs in IA-LPS BPD model rats. Other studies have shown that exosomes have similar therapeutic effects as MSCs. Future studies should consider comparing the effects of different EV subsets on experimental BPD models in a more direct way. Indeed, a single active ingredient has not been further investigated in this study. Rather, MVs likely provide an orchestra of bioactive components that function synergistically to play a therapeutic role. The reason why MVs retained cell proliferation but had no effect on cell death also needs further research. Furthermore, because IA-LPS BPD model focused on antenatal factors, the findings of this study need to be interpreted in the context of the experimental model. 


\section{Conclusion}

In conclusion, we demonstrated that MVs derived from hUCMSCs restored lung architecture and function and improved RVH in the IA-LPS BPD model by promoting AT2 cell proliferation and attenuating lung inflammation. The underlying mechanism was associated with the PTEN/AKT pathway and the MAPK pathway. Our findings may offer a new perspective for the treatment of BPD by MVs.

\section{Conflicts of interest}

The authors declare that they have no competing interests.

\section{Availability of data and material}

The authors confirm that all data underlying the findings are fully available.

\section{Funding}

This work was supported by the National Clinical Research Center for Child Health and Disorders (NCRC-2019-GP-11) and the Ministry of Science and Technology of China (2016YFA0101301).

\section{Acknowledgements}

We thank Professor Wei Shi (University of Southern California, CA, USA) for his assistance in our experiments.

\section{Note}

This manuscript has a preprint on https://www.researchsquare.com/article/rs$798865 / \mathrm{v} 1[51]$. 


\section{References}

1 Jobe AH. Animal Models, Learning Lessons to Prevent and Treat Neonatal Chronic Lung Disease [in eng]. Frontiers in medicine 2015;2:49.

2 Kinsella JP, Greenough A, Abman SH. Bronchopulmonary dysplasia [in eng]. Lancet (London, England) 2006;367(9520):1421-1431.

3 Stoll BJ, Hansen NI, Bell EF et al. Neonatal outcomes of extremely preterm infants from the NICHD Neonatal Research Network [in eng]. Pediatrics 2010;126(3):443-456.

4 Abman SH, Bancalari E, Jobe A. The Evolution of Bronchopulmonary Dysplasia after 50 Years [in eng]. American journal of respiratory and critical care medicine 2017;195(4):421-424.

5 Jensen EA, Schmidt B. Epidemiology of bronchopulmonary dysplasia [in eng]. Birth defects research Part A, Clinical and molecular teratology 2014;100(3):145-157. Northway WH, Rosan RC, Porter DY. Pulmonary disease following respirator therapy of hyaline-membrane disease. Bronchopulmonary dysplasia [in eng]. The New England journal of medicine 1967;276(7):357-368.

Higgins RD, Jobe AH, Koso-Thomas $\mathrm{M}$ et al. Bronchopulmonary Dysplasia: Executive Summary of a Workshop [in eng]. The Journal of pediatrics 2018;197:300-308. Ambalavanan N, Van Meurs KP, Perritt R et al. Predictors of death or bronchopulmonary dysplasia in preterm infants with respiratory failure [in eng]. J Perinatol 2008;28(6):420-426. Stoll BJ, Hansen NI, Bell EF et al. Trends in Care Practices, Morbidity, and Mortality of Extremely Preterm Neonates, 1993-2012 [in eng]. Jama 2015;314(10):1039-1051. 
cord blood Ureaplasma urealyticum and Mycoplasma hominis cultures in very preterm newborn infants [in eng]. American journal of obstetrics and gynecology 2008;198(1):43.e41-45. among infants born before the 28th week of gestation [in eng]. Pediatrics 2009;124(3):e450e458. disease: a 13-year hospital cohort study [in eng]. Pediatrics 2009;123(5):1314-1319. inflammation are associated with an increased risk of bronchopulmonary dysplasia [in eng]. Acta Paediatr 2015;104(3):259-263. Dysplasia and Late Respiratory Disease in Preterm Infants [in eng]. American journal of respiratory and critical care medicine 2017;196(3):364-374. With Bronchopulmonary Dysplasia Among Preterm Infants: A Systematic Review, Metaanalysis, and Metaregression [in eng]. JAMA network open 2019;2(11):e1914611. Preserves Lung Alveolar and Vascular Growth in Experimental BPD [in eng]. American journal of respiratory and critical care medicine 2020 . Inflammation and Hypoalveolarization in a Murine Model of Bronchopulmonary Dysplasia [in eng]. Frontiers in immunology 2020;11:579628. 

in preterm lamb lungs [in eng]. American journal of physiology Lung cellular and molecular physiology 2004;287(6):L1178-L1185.

Chou H-C, Li Y-T, Chen C-M. Human mesenchymal stem cells attenuate experimental bronchopulmonary dysplasia induced by perinatal inflammation and hyperoxia [in eng]. American journal of translational research 2016;8(2):342-353. from hyperoxic lung injury by ameliorating aberrant elastin remodeling in the lung of $\mathrm{O}(2)$ exposed newborn rat [in eng]. Biochem Biophys Res Commun 2018;495(2):1972-1979. Extracellular Vesicles Alleviate Lung Injury in Rat Model of Bronchopulmonary Dysplasia by Affecting Cell Survival and Angiogenesis. Stem Cells Dev 2020;29(23):1520-1532. Wilson JG, Liu KD, Zhuo H et al. Mesenchymal stem (stromal) cells for treatment of ARDS: a phase 1 clinical trial [in eng]. The Lancet Respiratory medicine 2015;3(1):24-32.

Chang YS, Ahn SY, Yoo HS et al. Mesenchymal stem cells for bronchopulmonary dysplasia: phase 1 dose-escalation clinical trial [in eng]. The Journal of pediatrics 2014;164(5):966972.e966. Ahn SY, Chang YS, Kim JH et al. Two-Year Follow-Up Outcomes of Premature Infants Enrolled in the Phase I Trial of Mesenchymal Stem Cells Transplantation for Bronchopulmonary Dysplasia [in eng]. The Journal of pediatrics 2017;185:49-54.e42. Ionescu L, Byrne RN, van Haaften T et al. Stem cell conditioned medium improves acute lung injury in mice: in vivo evidence for stem cell paracrine action [in eng]. American journal of 
physiology Lung cellular and molecular physiology 2012;303(11):L967-L977. attenuates experimental bronchopulmonary dysplasia in part via exosome-associated factor TSG-6. Stem Cell Res Ther 2018;9(1):173. mesenchymal stem cell-derived extracellular vesicles reduces lung injury in a rat model of bronchopulmonary dysplasia [in eng]. American journal of physiology Lung cellular and molecular physiology 2019;316(1):L6-119. Ameliorate Experimental Bronchopulmonary Dysplasia and Restore Lung Function through Macrophage Immunomodulation [in eng]. American journal of respiratory and critical care medicine 2018;197(1):104-116. and other extracellular vesicles for cell-to-cell communication [in eng]. Nature cell biology 2019;21(1):9-17.

Chen WX, Zhou J, Zhou SS et al. Microvesicles derived from human Wharton's jelly mesenchymal stem cells enhance autophagy and ameliorate acute lung injury via delivery of miR-100 [in eng]. Stem Cell Res Ther 2020;11(1):113. Ferreira ADF, Gomes DA. Stem Cell Extracellular Vesicles in Skin Repair [in eng]. Bioengineering (Basel, Switzerland) 2018;6(1). derived Microvesicles in Severe Pneumonia in Mice [in eng]. American journal of respiratory 
and critical care medicine 2015;192(3):324-336.

Kang T, Jones TM, Naddell C et al. Adipose-Derived Stem Cells Induce Angiogenesis via Microvesicle Transport of miRNA-31 [in eng]. Stem cells translational medicine 2016;5(4):440-450.

Wallace B, Peisl A, Seedorf G et al. Anti-sFlt-1 Therapy Preserves Lung Alveolar and Vascular Growth in Antenatal Models of Bronchopulmonary Dysplasia [in eng]. American journal of respiratory and critical care medicine 2018;197(6):776-787.

Mason RJ. Biology of alveolar type II cells [in eng]. Respirology (Carlton, Vic) 2006;11 Suppl:S12-15.

Ahn SY, Park WS, Kim YE et al. Vascular endothelial growth factor mediates the therapeutic efficacy of mesenchymal stem cell-derived extracellular vesicles against neonatal hyperoxic lung injury [in eng]. Experimental \& molecular medicine 2018;50(4):1-12.

Chimenti L, Luque T, Bonsignore MR et al. Pre-treatment with mesenchymal stem cells reduces ventilator-induced lung injury [in eng]. Eur Respir J 2012;40(4):939-948.

Velten M, Heyob KM, Rogers LK et al. Deficits in lung alveolarization and function after systemic maternal inflammation and neonatal hyperoxia exposure [in eng]. Journal of applied physiology (Bethesda, Md : 1985) 2010;108(5):1347-1356. matrix proteins and improves alveolarization in mice exposed to maternal inflammation and neonatal hyperoxia [in eng]. American journal of physiology Lung cellular and molecular physiology 2017;313(2):L339-1349. 
cell biology 2014;29:116-125.

Bobrie A, Colombo M, Raposo G et al. Exosome secretion: molecular mechanisms and roles in immune responses [in eng]. Traffic (Copenhagen, Denmark) 2011;12(12):1659-1668.

Kim CF, Jackson EL, Woolfenden AE et al. Identification of bronchioalveolar stem cells in normal lung and lung cancer [in eng]. Cell 2005;121(6):823-835.

Rawlins EL, Okubo T, Xue Y et al. The role of Scgb1a1+ Clara cells in the long-term maintenance and repair of lung airway, but not alveolar, epithelium [in eng]. Cell stem cell 2009;4(6):525-534.

Tropea KA, Leder E, Aslam M et al. Bronchioalveolar stem cells increase after mesenchymal stromal cell treatment in a mouse model of bronchopulmonary dysplasia [in eng]. American journal of physiology Lung cellular and molecular physiology 2012;302(9):L829-837. Hu Y, Wei X, Liao Z et al. Transcriptome Analysis Provides Insights into the Markers of Resting and LPS-Activated Macrophages in Grass Carp (Ctenopharyngodon idella) [in eng]. International journal of molecular sciences 2018;19(11). Willis GR, Fernandez-Gonzalez A, Reis M et al. Mesenchymal stromal cell-derived small extracellular vesicles restore lung architecture and improve exercise capacity in a model of neonatal hyperoxia-induced lung injury [in eng]. Journal of extracellular vesicles 2020;9(1):1790874. progenitor type II cells by using PTEN to orchestrate the PI3K/Akt signaling [in eng]. Stem Cell Res Ther 2017;8(1):138.

Kallapur SG, Moss TJ, Ikegami M et al. Recruited inflammatory cells mediate endotoxin- 
induced lung maturation in preterm fetal lambs [in eng]. American journal of respiratory and critical care medicine 2005;172(10):1315-1321.

49 Reinhardt J, Landsberg J, Schmid-Burgk JL et al. MAPK Signaling and Inflammation Link Melanoma Phenotype Switching to Induction of CD73 during Immunotherapy [in eng]. Cancer research 2017;77(17):4697-4709.

50 Harding A, Cortez-Toledo E, Magner NL et al. Highly Efficient Differentiation of Endothelial Cells from Pluripotent Stem Cells Requires the MAPK and the PI3K Pathways [in eng]. Stem Cells 2017;35(4):909-919.

51 Ou Zhou, Jingyi You, Xiaochuan Xu et al. Microvesicles derived from human umbilical cord mesenchymal stem cells enhance alveolar development and attenuate lung inflammation in a rat model of bronchopulmonary dysplasia induced by antenatal lipopolysaccharide. Research Square (preprint). 2021 DOI: 10.21203/rs.3.rs-798865/v1. 


\section{Figure Legend}

Figure 1: Establishment and characterization of bronchopulmonary dysplasia (BPD) model induced by intra-amniotic injection of LPS (IA-LPS). A. The schedule involved experimental BPD rat model caused by intra-amniotic (IA) injection of normal saline (NS) or LPS and time points to collect lungs. B. Representative lung sections stained with H\&E on postnatal day 1 (PN1), PN7, and PN14, scale bar $=100 \mu \mathrm{m}$. Quantification of the mean linear intercept (MLI, C) and secondary septa (D). ( $N=3$, t-test, NS: not significant, $* * * P<0.001)$.

Figure 2: hUCMSCs treatment attenuates lung morphology and function in the IA-LPS BPD model. A. The study design represents the IA-LPS BPD model and hUCMSCs treatment. Transtracheal administration of hUCMSCs was conducted on PN7. Study end points on PN14 involved analysis of lung morphology, function, and right ventricular hypertrophy (RVH). B. Representative lung sections stained with H\&E on PN14, scale bar $=200 \mu \mathrm{m}$. C. Quantification of MLI. $(N=5$, ANOVA, $* * * P<0.001)$. D. Quantification of radial alveolar counts (RAC). $(N=5$, ANOVA, $* * * P<0.001)$. E. Comparison of pups' weight among four groups. ( $N=5$, ANOVA). F. Representative immunofluorescence images of vWF staining in the lung on PN14 in each group, scale bar $=100 \mu \mathrm{m}$. G. Quantification of vWF-positive vessels $(<100 \mu \mathrm{m}) .(N=5$, ANOVA, $* * P<0.01, * * * P<0.001)$. Lung resistance (LR, H) and dynamic compliance (Cdyn, I) were determined from anesthetized and ventilated pups. $(N=5$, ANOVA, $* * * P<0.001)$. J. The right ventricular index was determined by $\mathrm{RV} / \mathrm{LV}+\mathrm{S}$ to measure $\mathrm{RVH}$. $(N=5$, ANOVA, $* * * P<0.001)$ 
Figure 3: Characterization of hUCMSCs-derived MVs. A. Representative images of transmission electron microscopy (TEM) for MVs derived from hUCMSCs, scale bar $=500 \mathrm{~nm}$. B. Detection of MVs-specific biomarkers for both analyzed MVs and wholecell lysates by western blot. C. Nanoparticle tracking analysis (NTA) measurement of the mean size of MVs. D. Representative images showing the co-localization of phalloidin immunostaining (green) with internalized MVs (red), scale bar $=50 \mu \mathrm{m} . \mathbf{E}$. MLE-12 viability after exposure to different concentrations of LPS in vitro. ( $N=3$, ANOVA, $* P<0.05, * * * P<0.001)$.

Figure 4: MVs improve antenatal LPS-induced lung injury in a dose-dependent manner. A. Representative lung sections stained with $\mathrm{H} \& \mathrm{E}$ on PN14, scale bar $=100 \mu \mathrm{m}$. B. Quantification of the MLI. ( $N=3-4$, ANOVA, NS: not significant, $* * * P<0.001)$.

Figure 5: MVs improve lung development and function in the IA-LPS BPD model. A. Representative lung sections stained with H\&E on PN14, scale bar $=200 \mu \mathrm{m}$. B. Quantification of MLI. $(N=5$, ANOVA, $* * * P<0.001)$. C. Quantification of RAC. $(N$ $=5$, ANOVA, $* * * P<0.001)$. D. Comparison of pups' weight among four groups. $(N=$ 5, ANOVA). E. Representative immunofluorescence images of vWF staining in the lung on PN14 in each group, scale bar $=100 \mu \mathrm{m}$. F. Quantification of vWF-positive vessels $(<100 \mu \mathrm{m}) .(N=5$, ANOVA, NS: not significant, $* * * P<0.001)$. Lung resistance (LR, G) and dynamic compliance (Cdyn, H) were determined from anesthetized and ventilated pups. $(N=5$, ANOVA, $* * * P<0.001)$. I. RV/LV $+\mathrm{S}$ to measure RVH. $(N=$ 5, ANOVA, $* * * P<0.001)$.

Figure 6: Location of the transtracheally delivered MVs according to the lung tissue 
cell types. A. Representative immunofluorescence images of donor MVs and host lung cells staining at 48 hours after MVs administration. The MVs were pre-stained with DiO dye (green). The nuclei were labeled with DAPI (blue); the AT1, AT2, vascular endothelial cells, total macrophages, vascular smooth muscle cells, and vascular pericytes were stained with AQP1, SP-C, CD31, Iba-1, $\alpha$-SMA, and NG2 (red), respectively. B. The rate of donor MVs incorporation into each type of host lung cells. $(N=5$, ANOVA, NS: not significant, $* * P<0.01, * * * P<0.001)$.

Figure 7: Effect of MVs on AT2 cells and macrophage-related inflammatory factors in vivo. A. Representative immunofluorescence images of SP-C (red) staining in lung, scale bar $=100 \mu \mathrm{m}$. B. Quantification of SP-C-positive cells in each group. $(N=5$, ANOVA, $\left.{ }^{* * *} P<0.001\right)$. C. Western blot detection of protein levels of SP-C in each group. D. Densitometric analysis was used to quantify the protein levels of SP-C. ( $N=$ 3, ANOVA, $* * * P<0.001)$. The levels of IL-6 (E) and IL-10 (F) in lung homogenate were measured with $\operatorname{ELISA}(N=3, \mathrm{ANOVA}, * * P<0.01, * * * P<0.001)$.

Figure 8: MVs improve MLE-12 cells proliferation following LPS-induced injury. A. Representative immunofluorescence images stained by Ki-67 (red) in MLE-12 cells, scale bar $=100 \mu \mathrm{m}$. B. Representative images of flow cytometric analysis of MLE-12 cell apoptosis for each group. C. Quantitative analysis of Ki-67-positive cells in MLE12 cells treated by LPS with or without MVs for 48 hours. $(N=5$, ANOVA, *** $P<$ 0.001). D. Quantitative analysis of the apoptotic rate of MLE-12 cells in each group. $(N=5$, ANOVA, NS: not significant, $* * * P<0.001)$. E. CCK-8 analysis of MLE-12 cells' viability. ( $N=5$, ANOVA). 
Figure 9: MVs activate the PTEN/p-AKT pathway and suppress the MAPK pathway in the IA-LPS BPD model. A. The protein levels of PTEN, p-AKT at S473, and AKT were evaluated by western blotting on lung homogenates on PN14. B. Densitometric analysis was used to quantify the expression of PTEN and p-AKT/AKT. $(N=3$, ANOVA, $\left.{ }^{* *} P<0.01, * * * P<0.001\right)$. C. The protein levels of $\mathrm{p} 38, \mathrm{JNK}, \mathrm{ERK}$, and their phosphorylated forms (p-p38, p-JNK, and p-ERK) were evaluated by western blotting on lung homogenates on PN14. D. Densitometric analysis was used to quantify the phosphorylation of p38 at Thr180/Tyr182, JNK at Thr183/Tyr185, and ERK1/2 at Thr202/Tyr1204. $\left(N=3\right.$, ANOVA, $\left.{ }^{* *} P<0.01,{ }^{* * *} P<0.001\right)$. E. The protein levels of VEGF-A in lung homogenate were evaluated by western blotting on PN14. F. Densitometric analysis was used to quantify the expression of VEGF-A. ( $N=3$, ANOVA, NS: not significant, $* * * P<0.001)$.

Figure S1: In vivo imaging of MVs in the IA-LPS BPD model. A. Representative fluorescence images of IA-LPS BPD rats after treatment with MVs at selected time points, scale bar $=10 \mu \mathrm{m}$. B. The number of DiO-positive cells in the lungs subjected to transtracheal injection of MVs at selected time intervals. $(N=5$, ANOVA, NS: not significant, $* * * P<0.001)$.

Figure S2: Effect of MVs on pulmonary surfactants. A. Western blot detection of protein levels of SP-A1, SP-B, and SP-D in each group. B. Densitometric analysis was used to quantify the protein levels of SP-A1, SP-B, and SP-D in each group. $(N=3$, ANOVA, NS: not significant, $* * * P<0.001)$.

Figure S3: Effect of MVs on lung macrophage infiltration. A. Representative 
immunofluorescence images of Iba-1 (red) staining in lung tissue. B. Quantification of Iba-1-positive cells in each group. $(N=5$, ANOVA, $* * * P<0.001)$. 


\section{Figure 1}

A

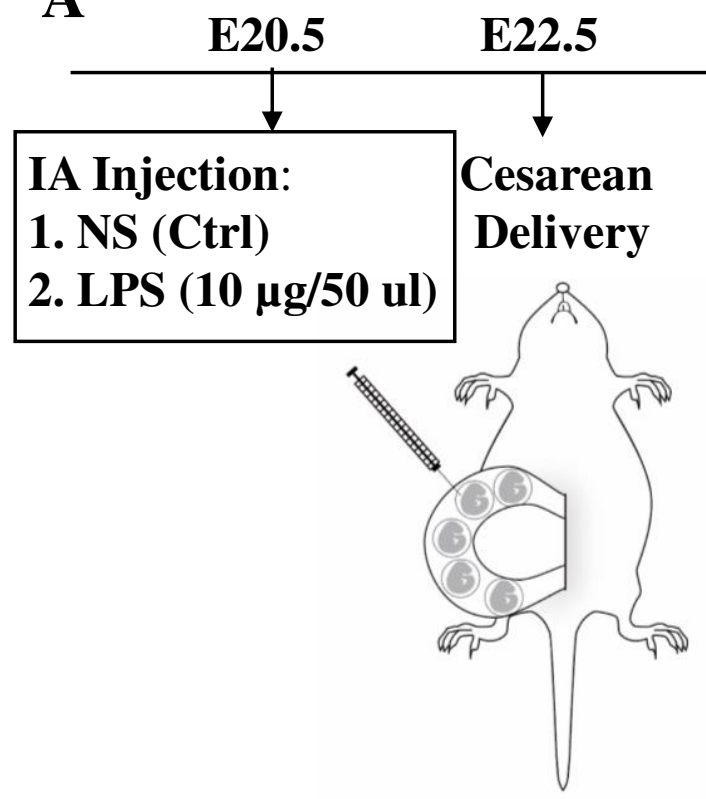

B

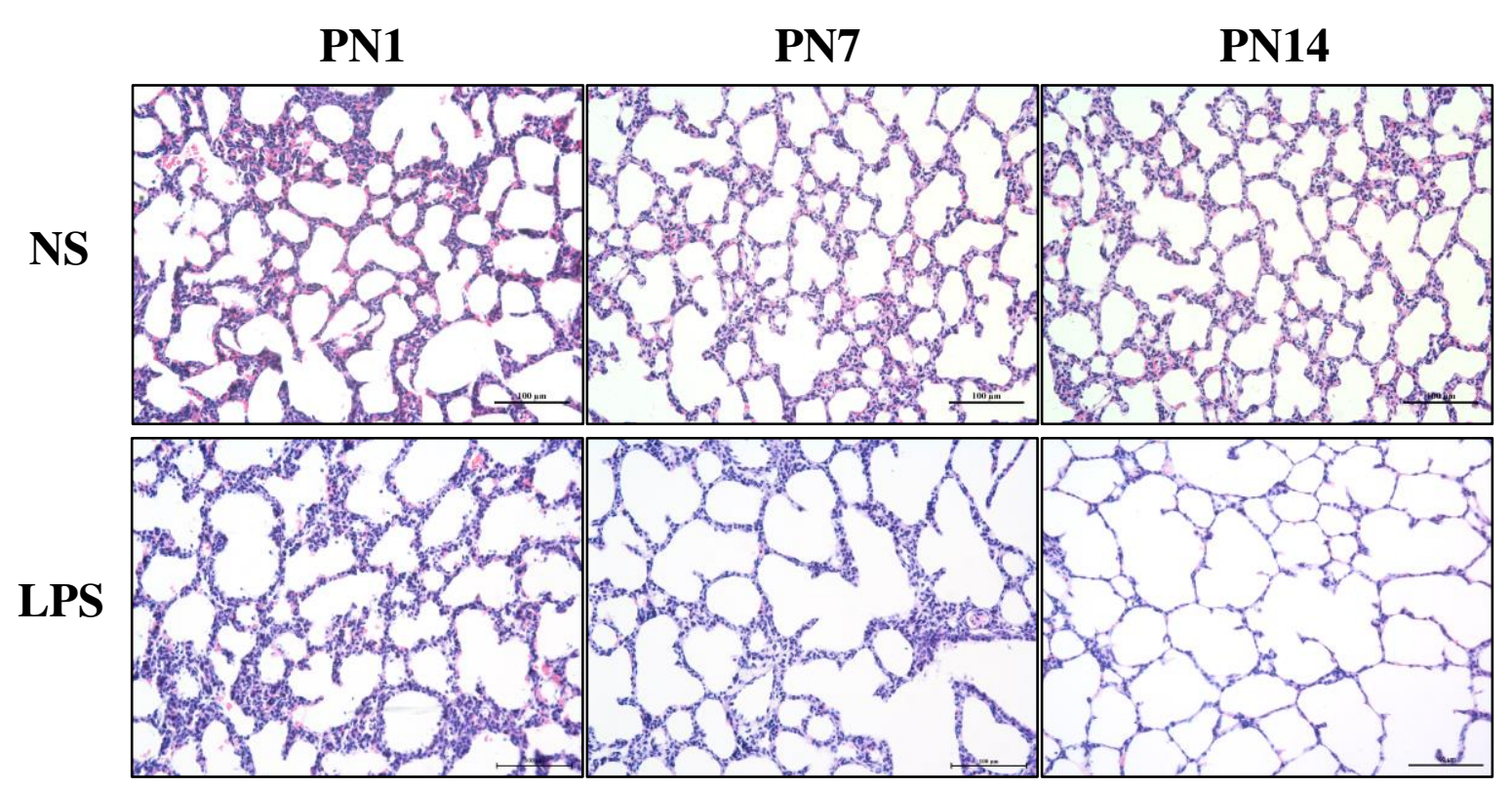

C

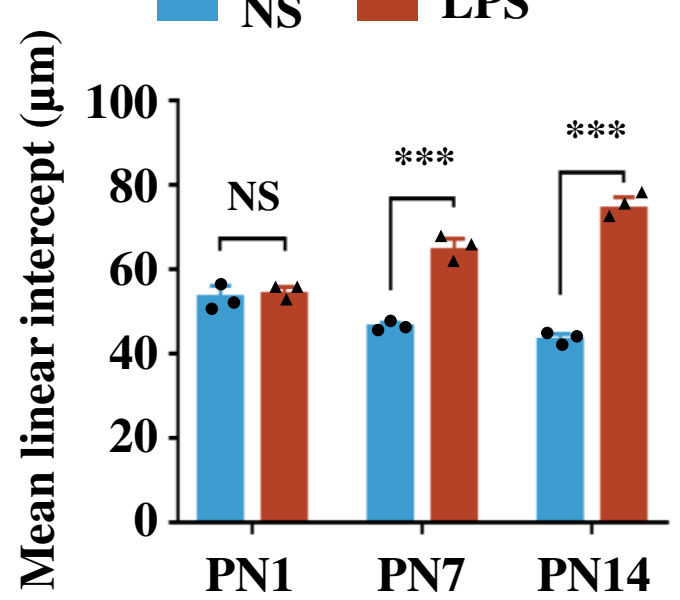

D

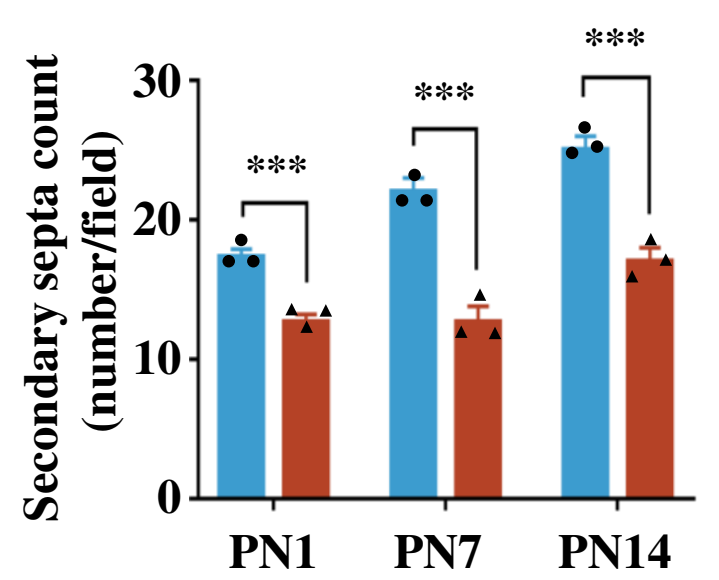




\section{Figure 2}

A

\begin{tabular}{|c|c|c|}
\hline E22.5 & PN7 & PN14 \\
\hline I & $\downarrow$ & $\downarrow$ \\
\hline IA-LPS BPD Model & $\begin{array}{l}\text { Transtracheal } \\
\text { Administration: } \\
\text { (1) NS (Ctrl) } \\
\text { (2) MSCs }\end{array}$ & $\begin{array}{l}\text { Study Endpoint : } \\
\text { (1) Lung morphology } \\
\text { (2) Lung function } \\
\text { (3) RVH }\end{array}$ \\
\hline
\end{tabular}

B

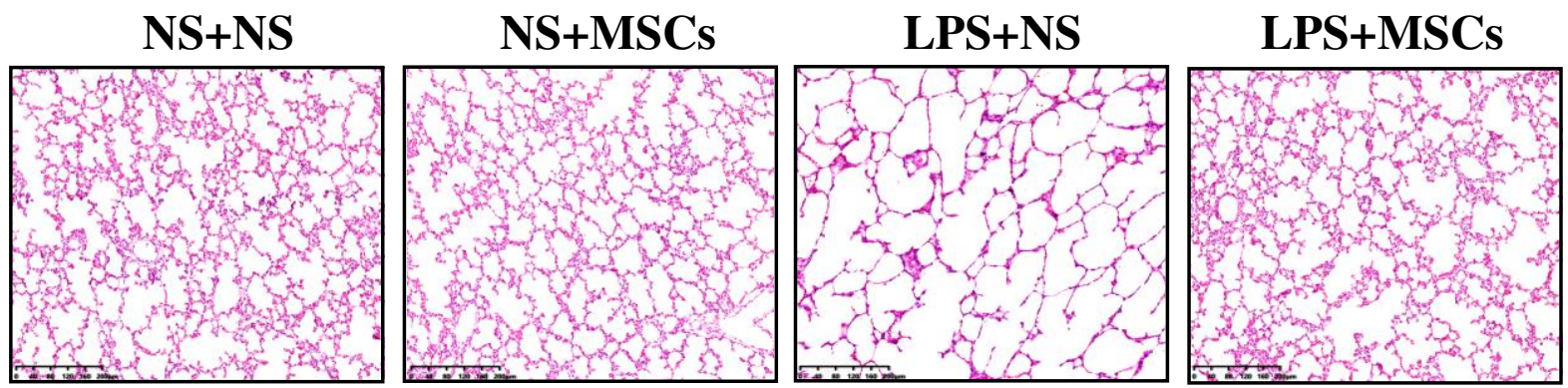

\begin{tabular}{llll}
$C$ & NS+NS & LPS+NS & D \\
\cline { 2 - 4 } & NS+MSCs & LPS+MSCs
\end{tabular}

E
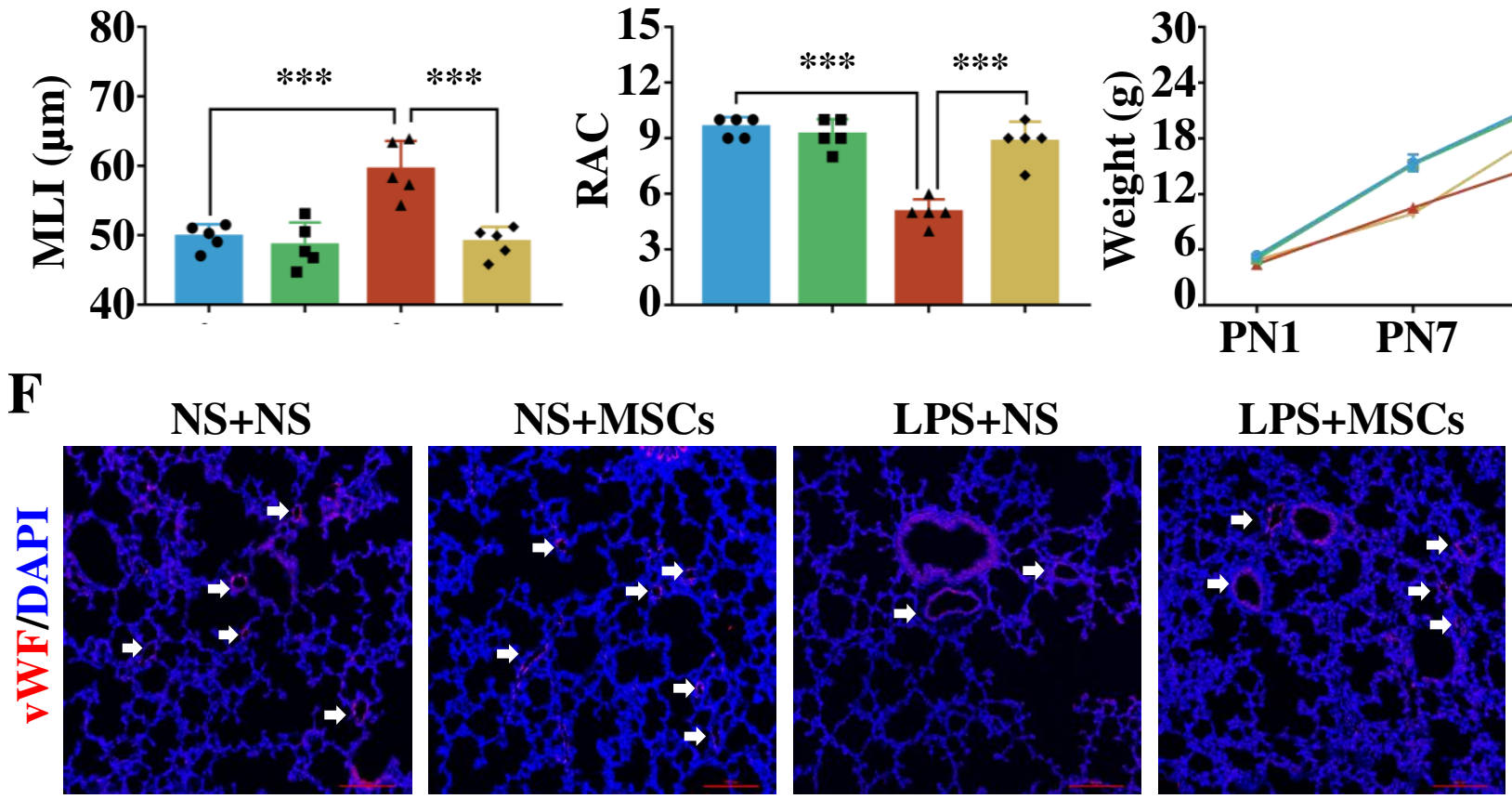

G

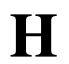

I

J
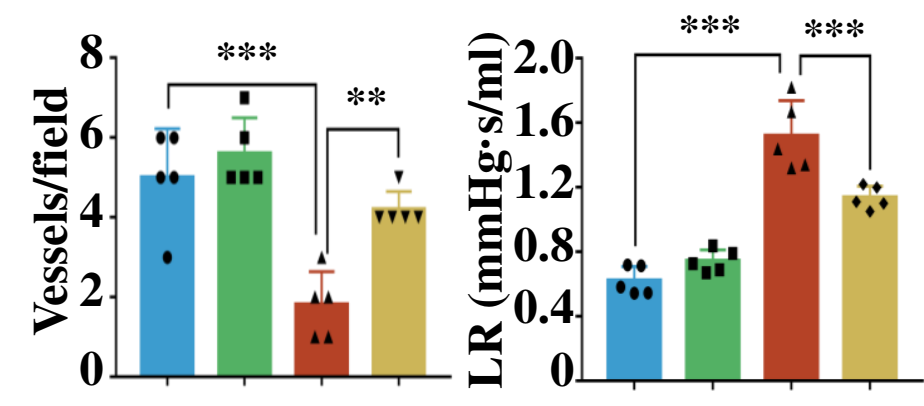

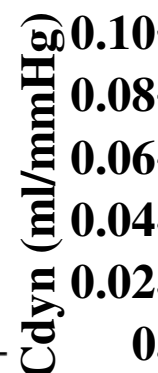

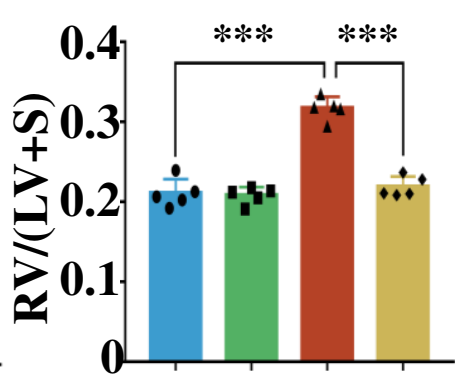




\section{Figure 3}

A
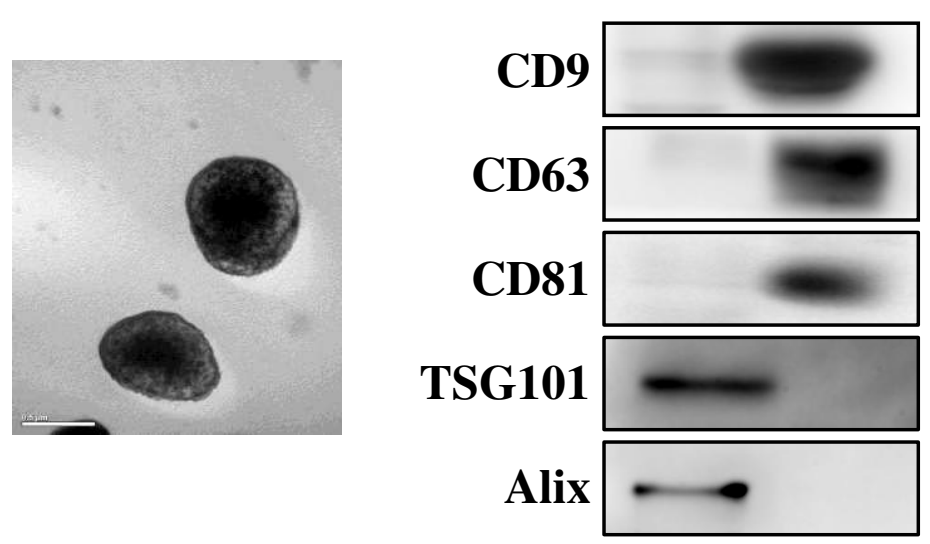

C

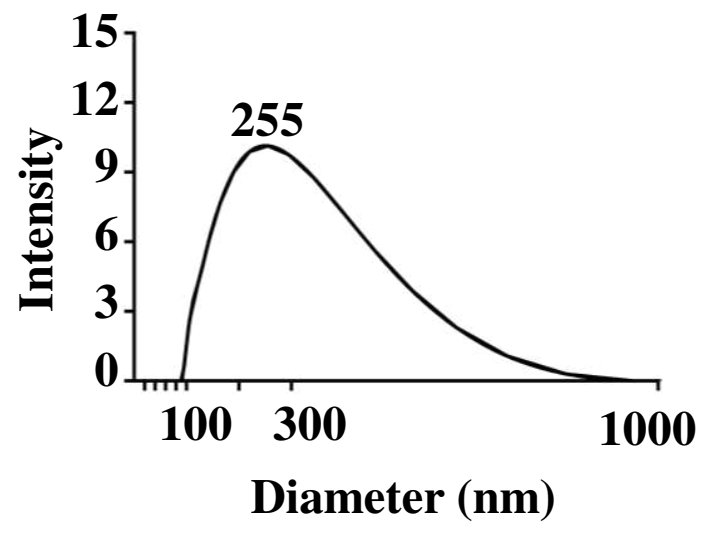

D
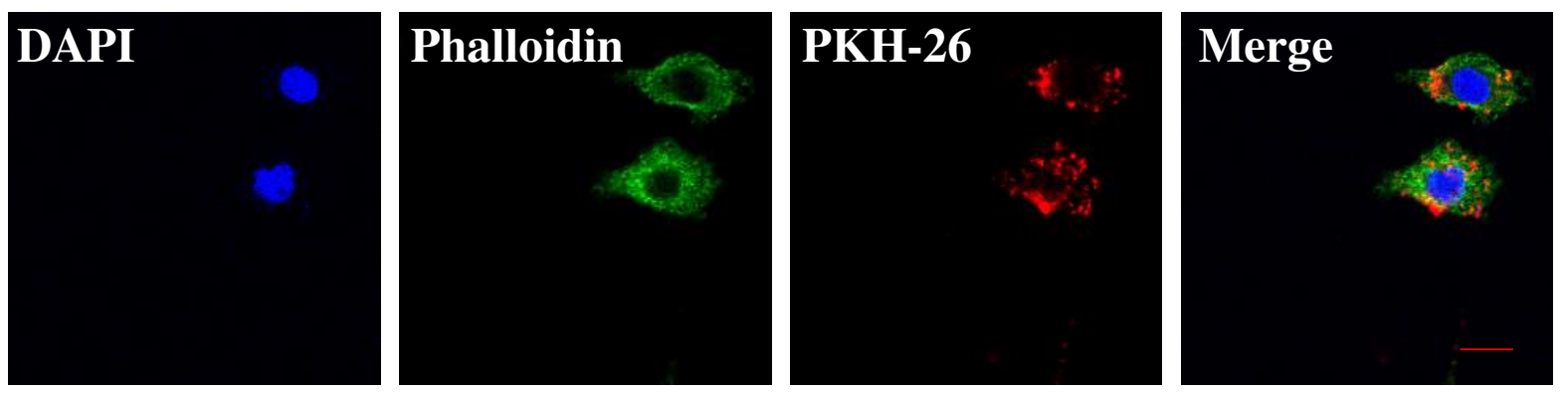

E

***

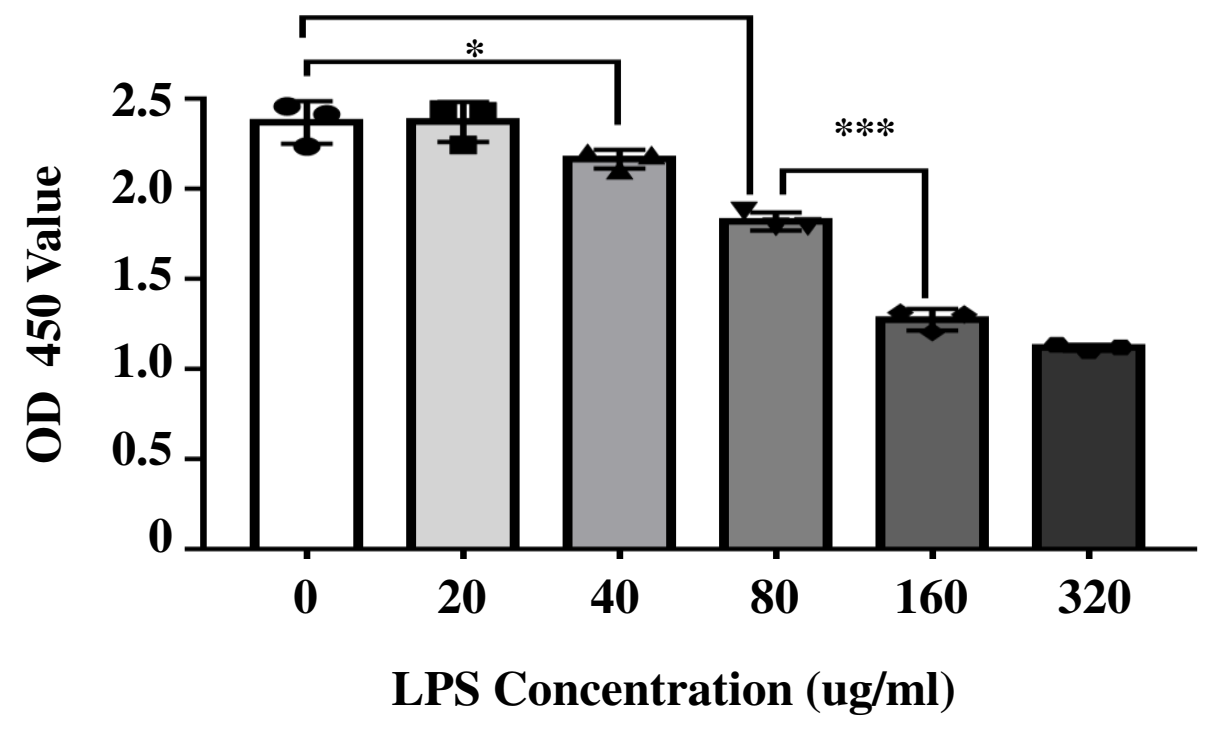




\section{Figure 4}

A

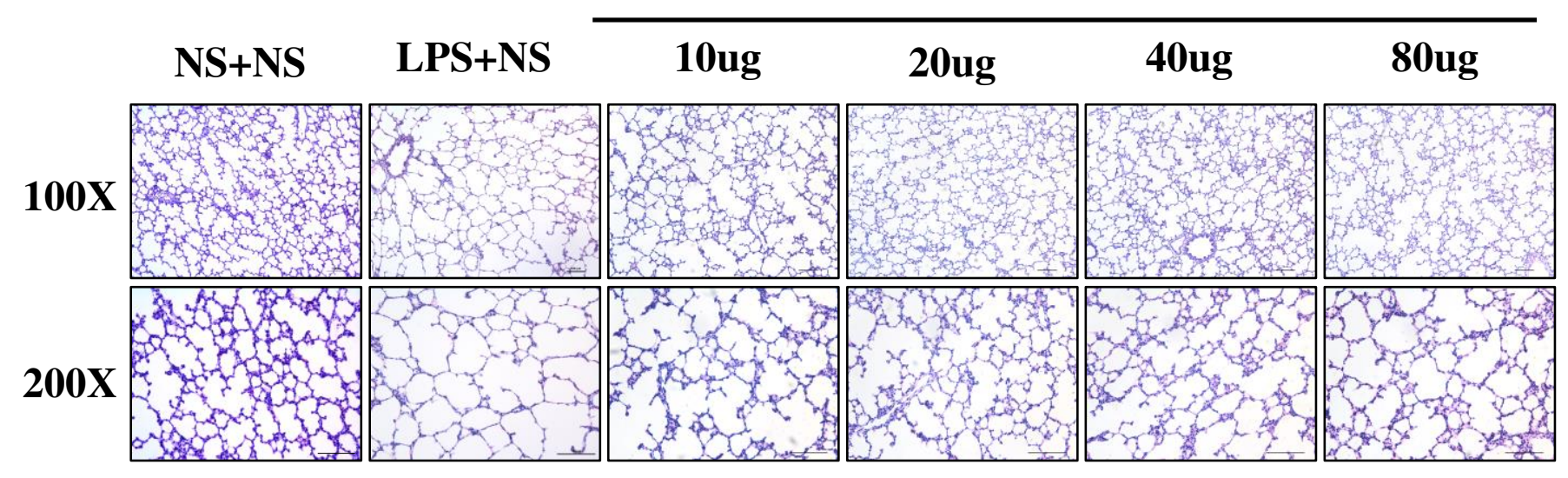

B

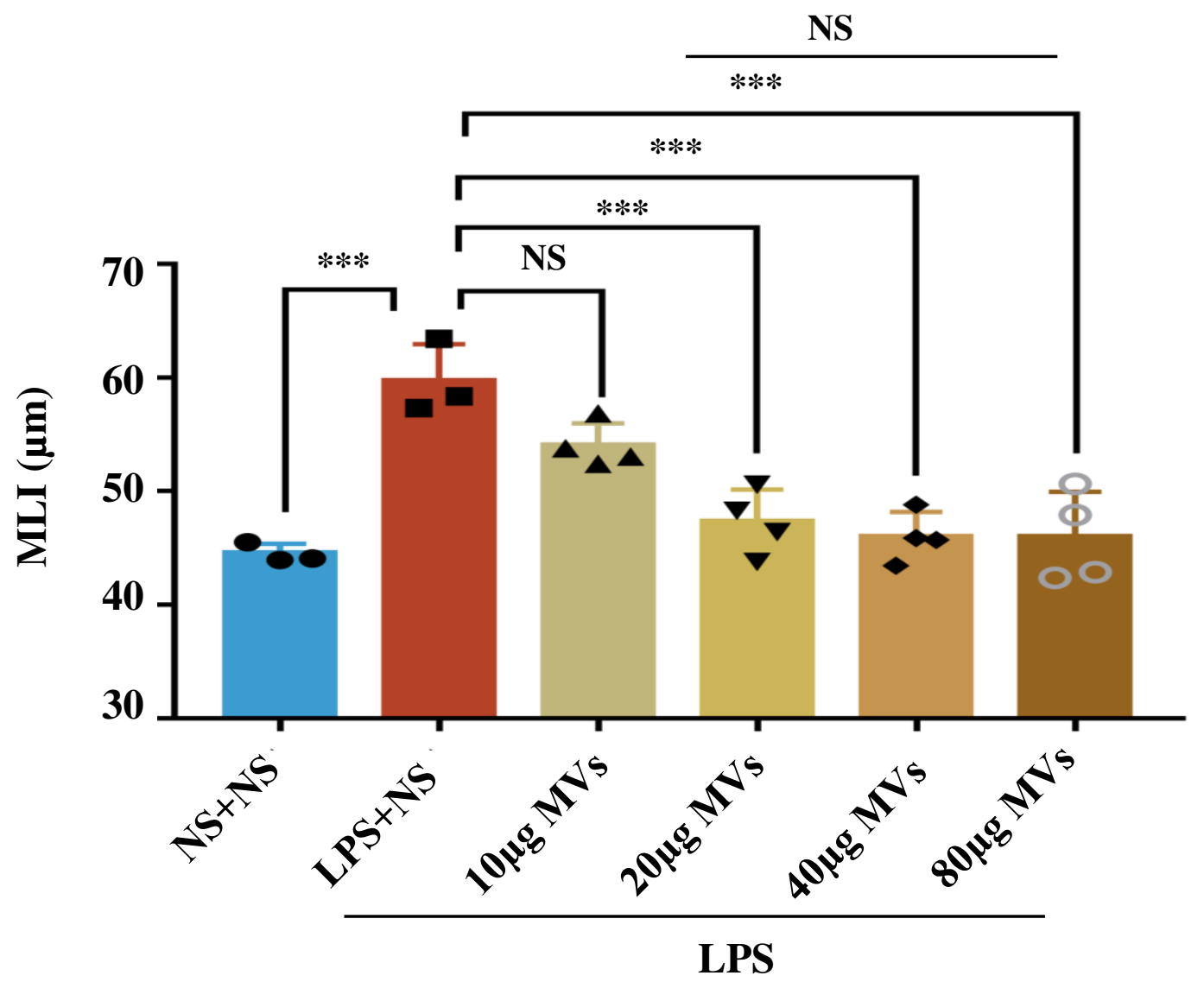




\section{Figure 5}

A
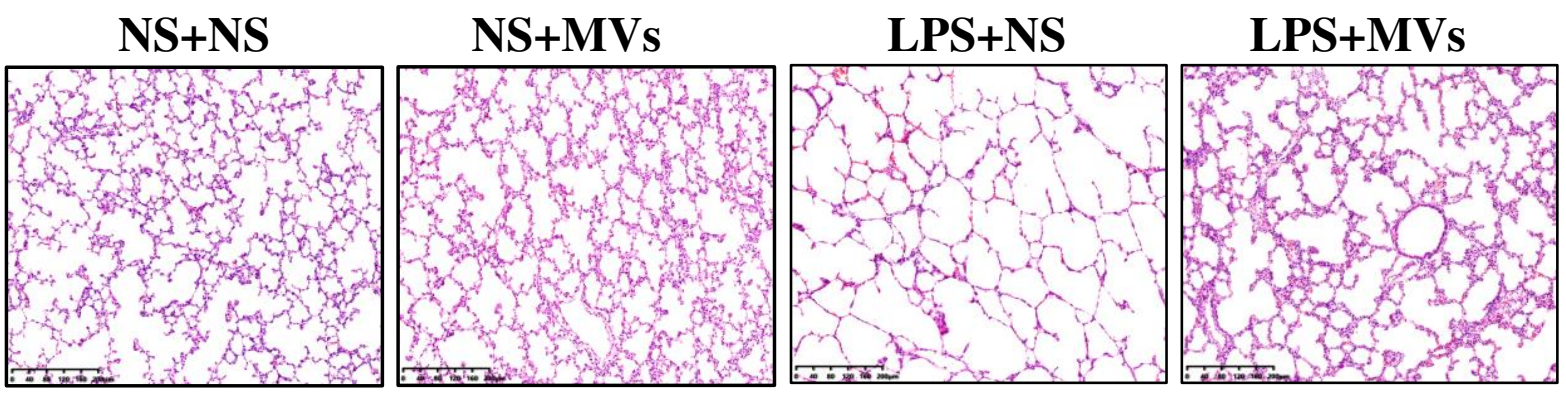

B

$\begin{array}{lll}\text { NS+NS } & \text { LPS+NS C } \\ \text { NS+MVs } & \text { LPS+MVs }\end{array}$

D
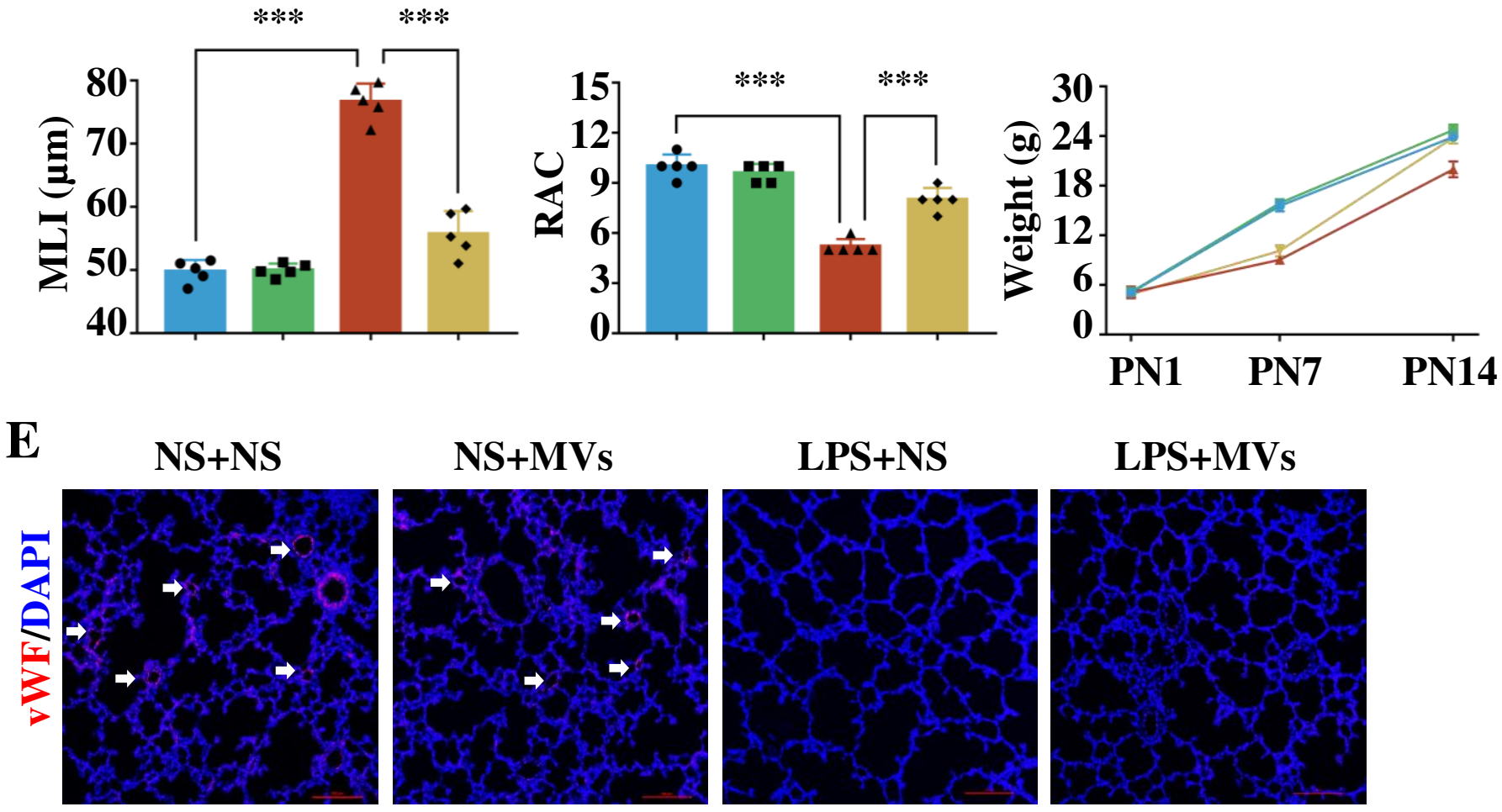

LPS+MVs

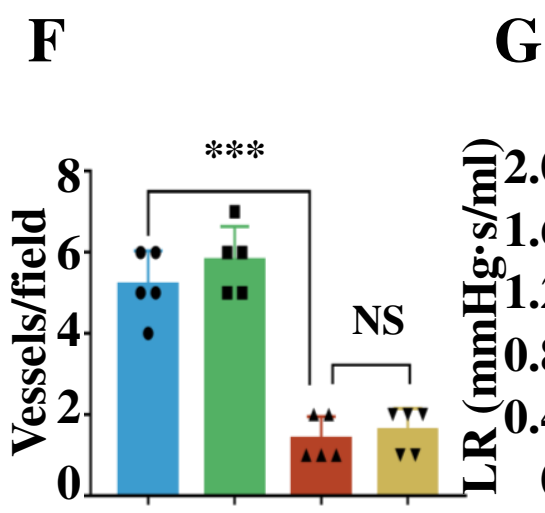

H

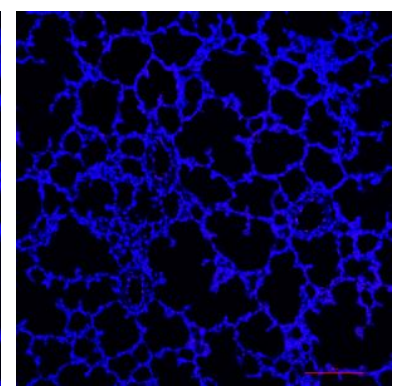

G

I

$* * \quad * * *$ 


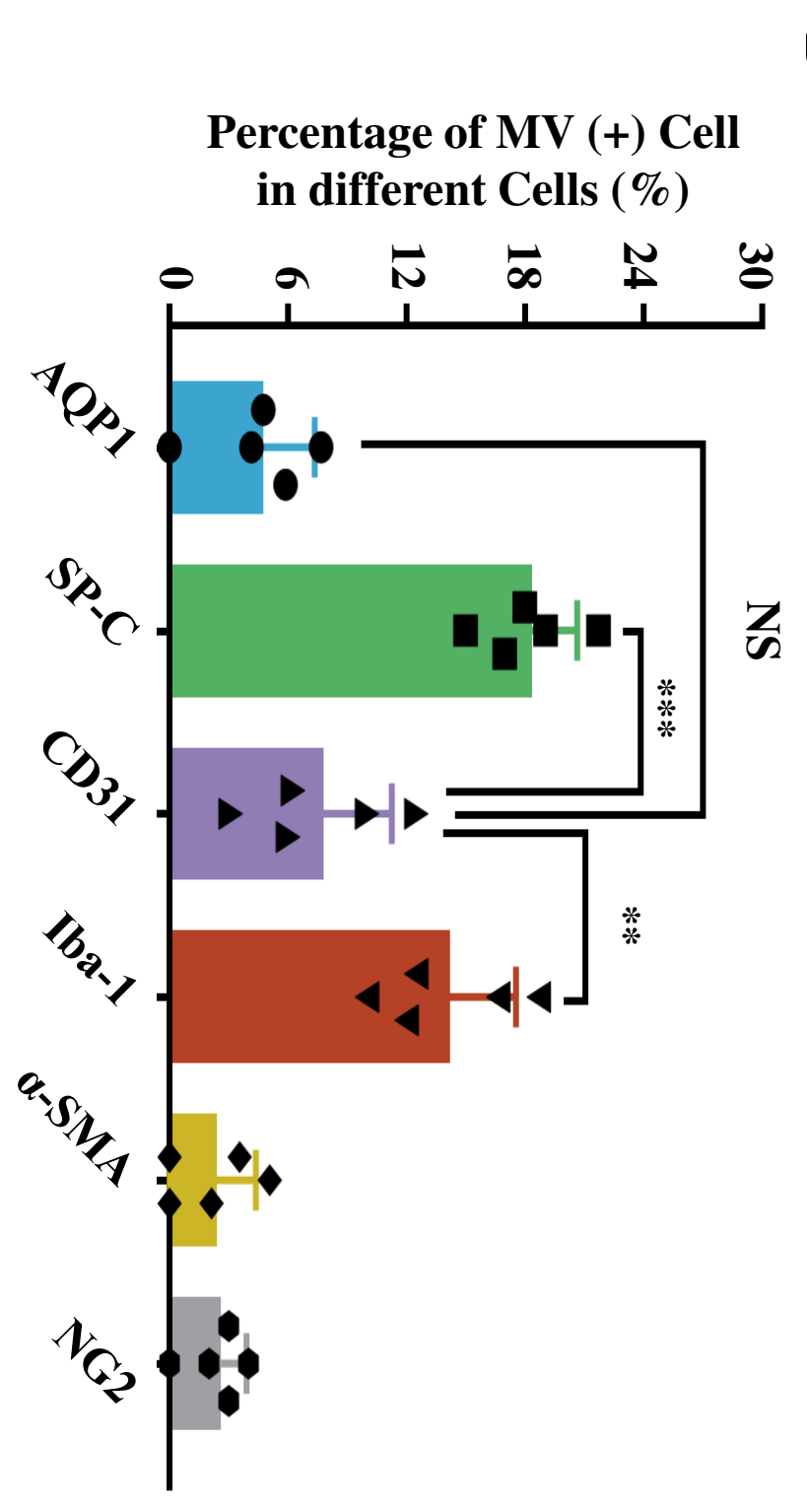

ש CD31/MVs/DAPI SP-C/MVs/DAPI AQP1/MVs/DAPI D
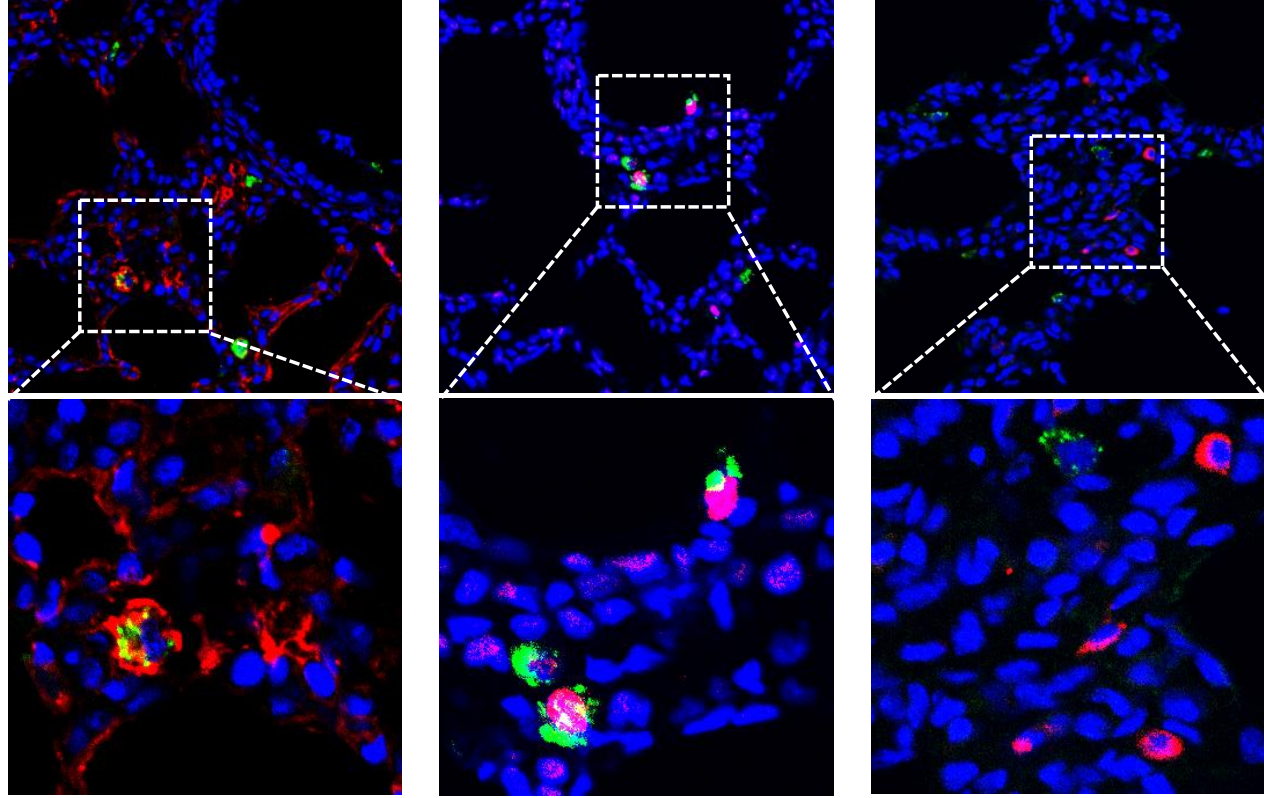

NG2 /MVs/DAPI $\alpha$-SMA/MVs/DAPI Iba-1/MVs/DAPI
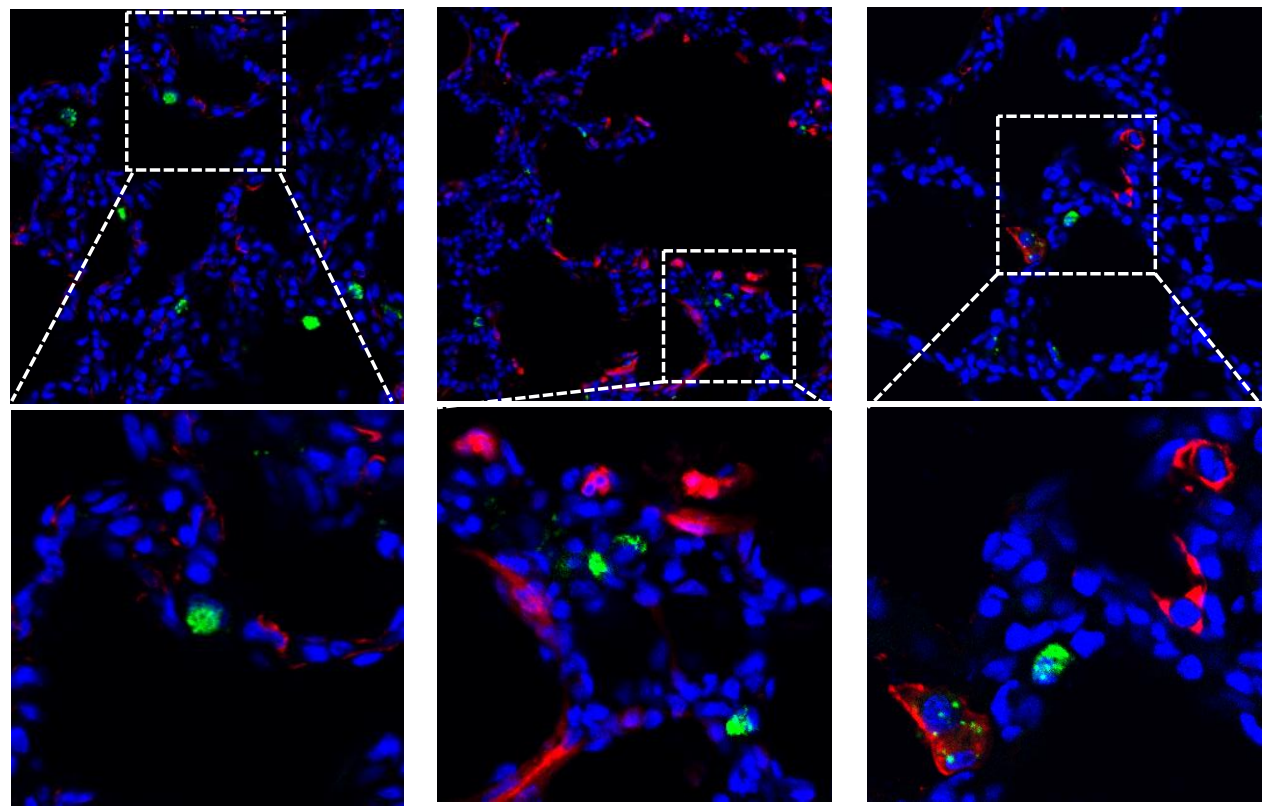

בְ. 


\section{Figure 7}

A

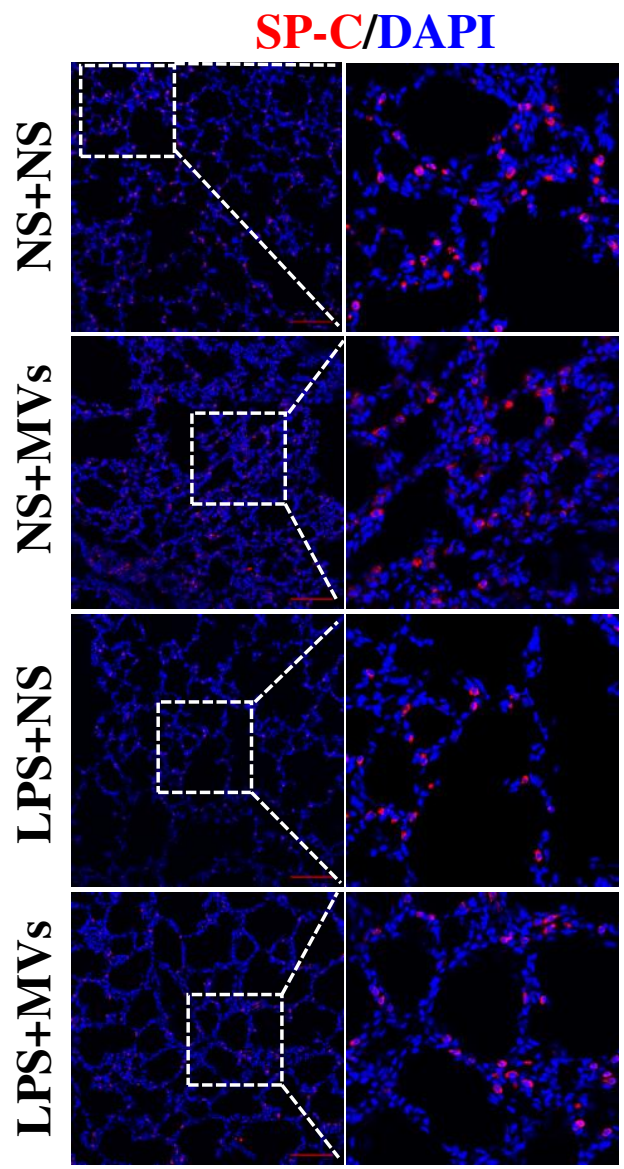

C

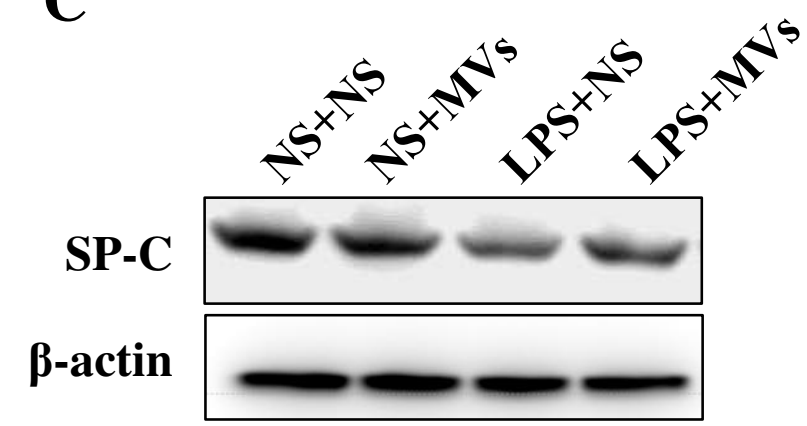

E

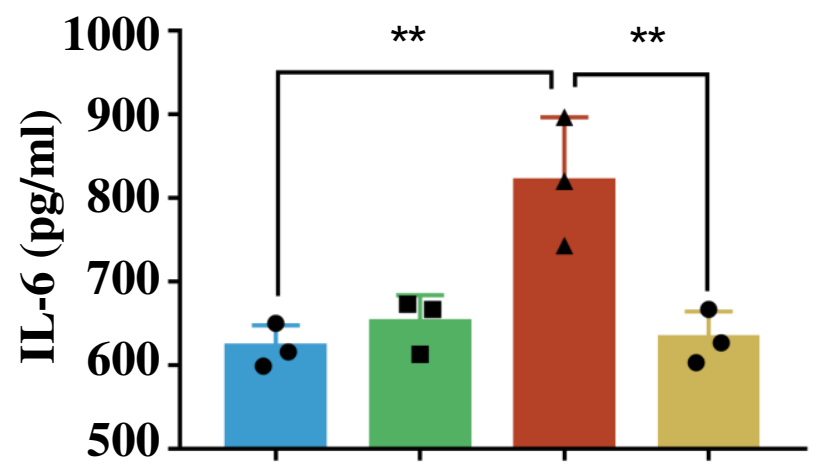

B
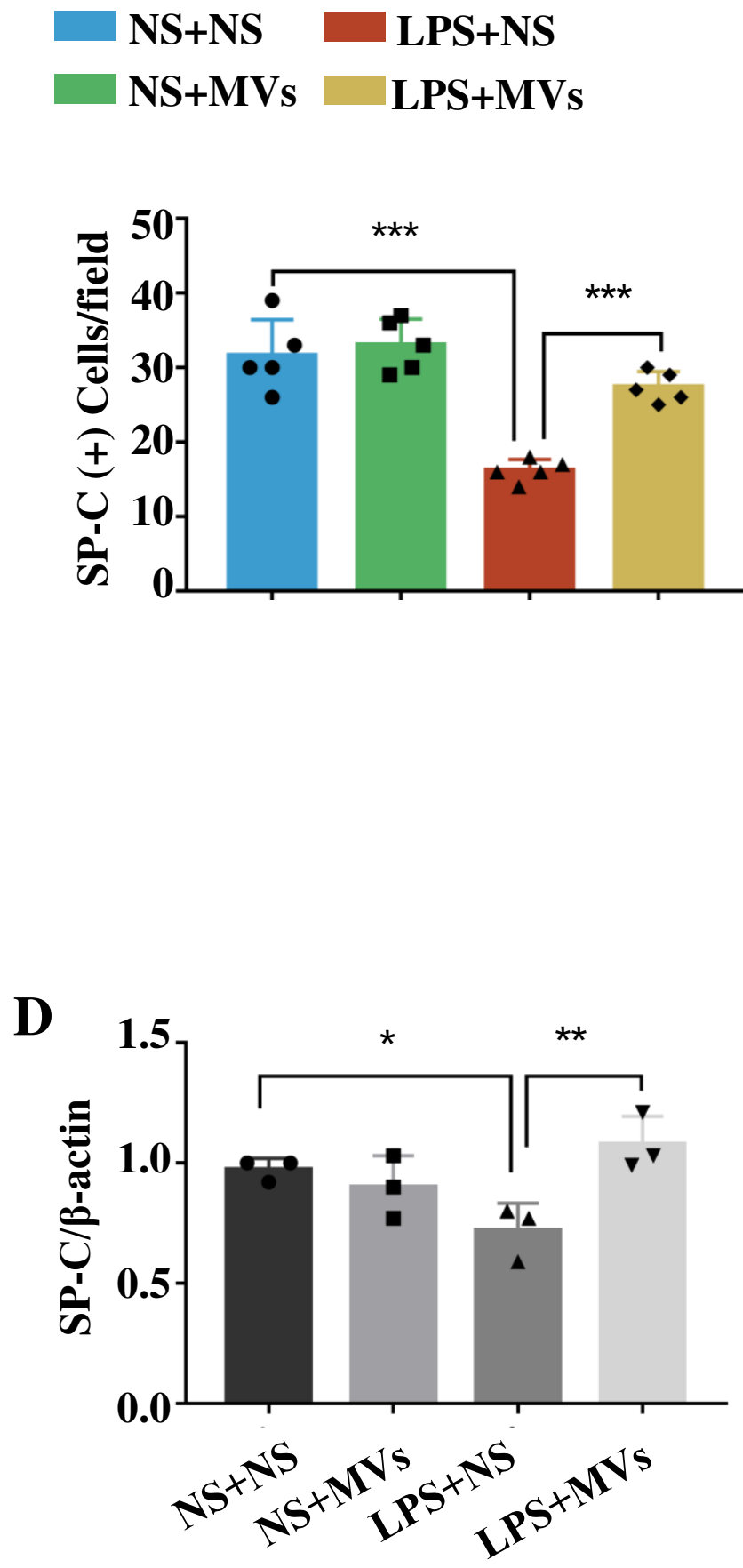

F

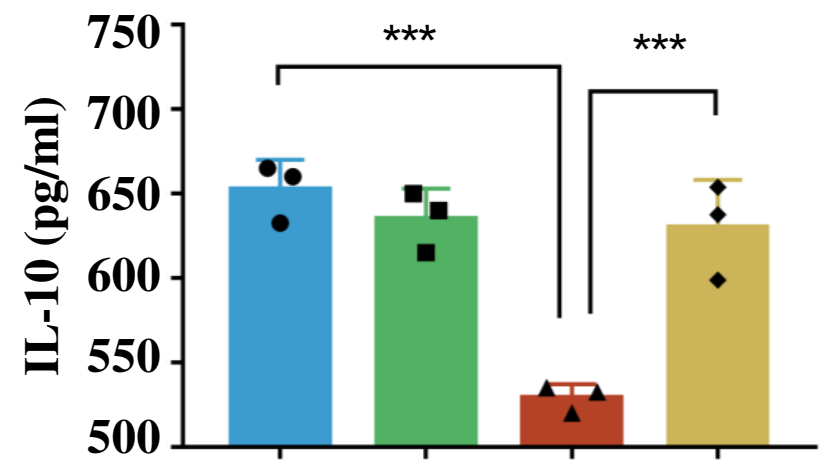




\section{Figure 8}
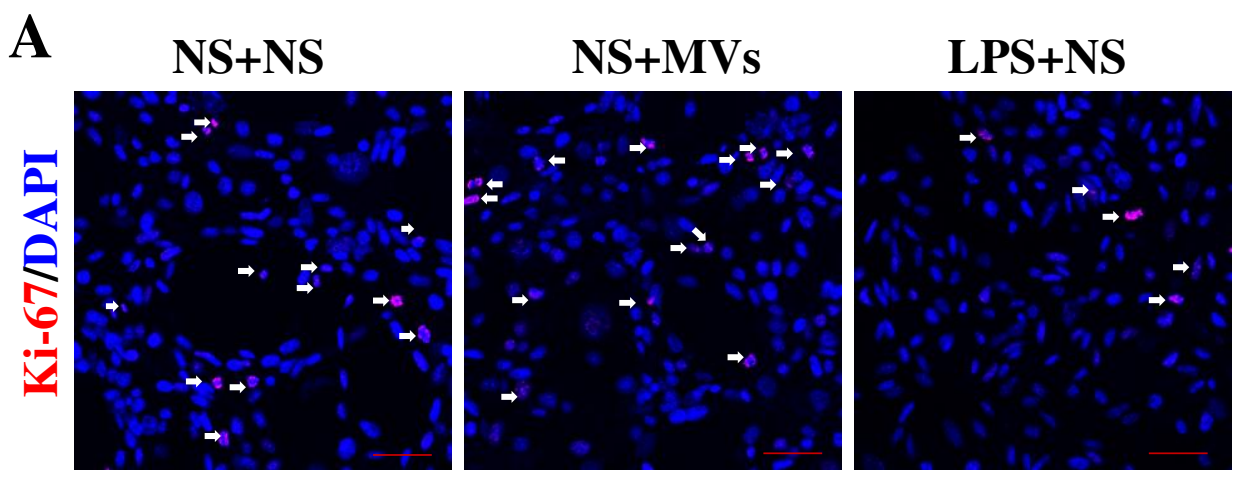

LPS+MVs

B

$\begin{array}{ll}\text { NS+NS } & \text { LPS+NS } \\ \text { NS+MVs } & \text { LPS+MVs }\end{array}$

C

NS+MVs LPS+MVs
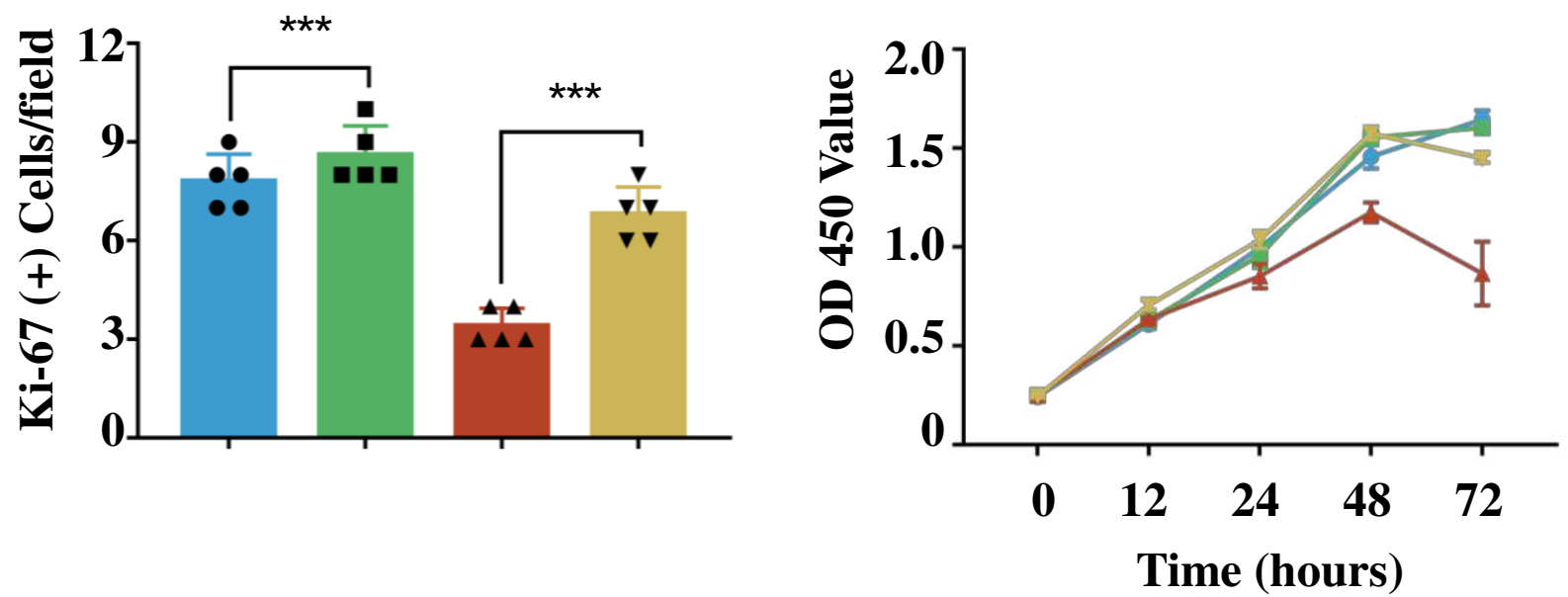
A

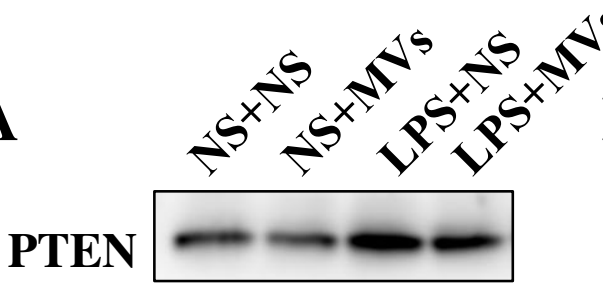

Figure 9

\section{B}

NS+NS LPS+NS

NS+MVs LPS+MVs

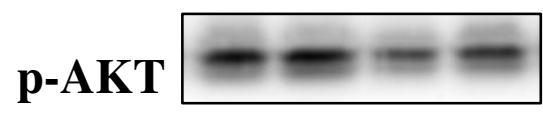

AKT

$\beta$-actin
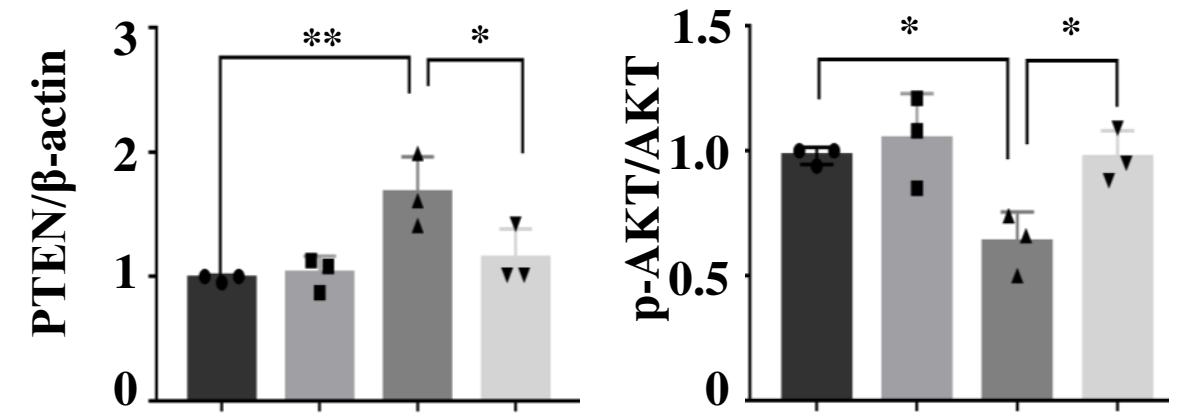

C
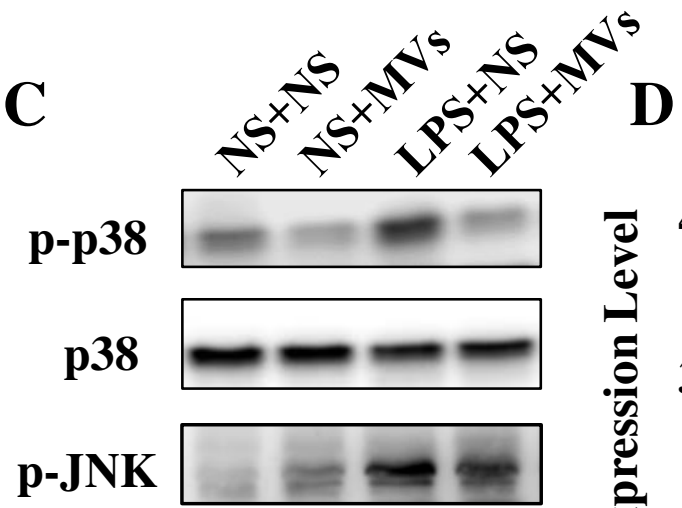

อุ

JNK $=-2=$
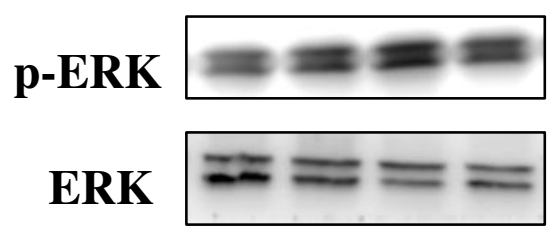

$\beta$-actin
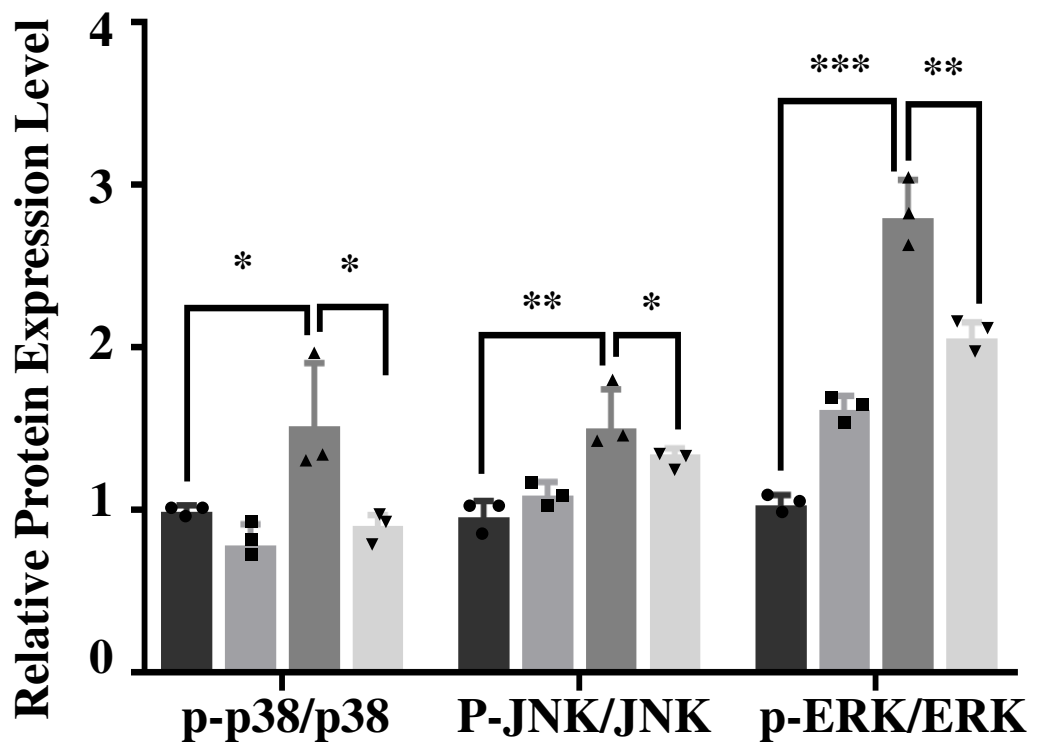

$\mathbf{E}$

VEGF-A

$\beta$-actin

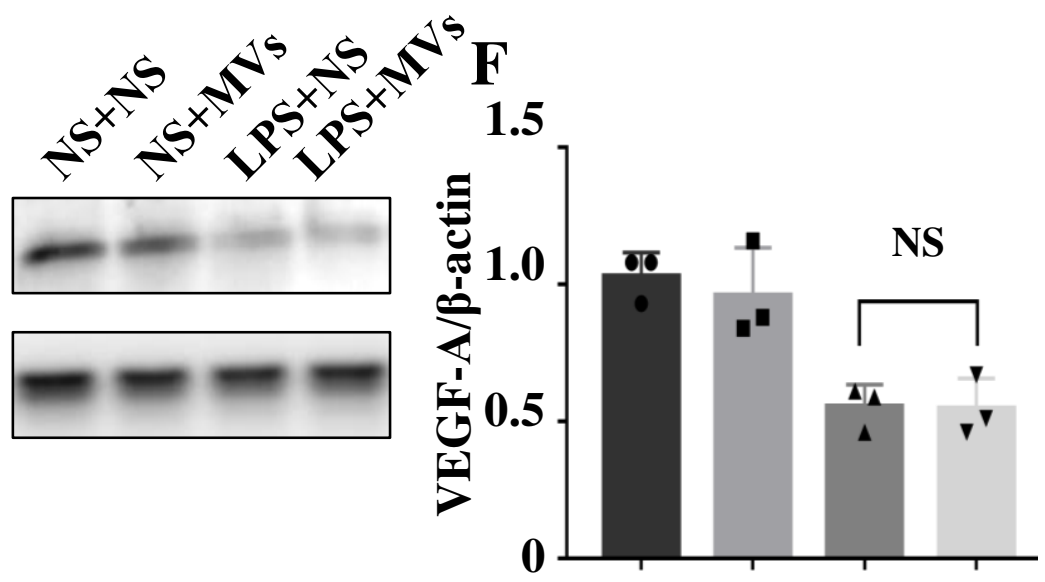




\section{Supplementary Files}

This is a list of supplementary files associated with this preprint. Click to download.

- supplementfigure.pdf 